\title{
Comparative Subsequence Sets Analysis (CoSSA) is a robust approach to identify haplotype specific SNPs; mapping and pedigree analysis of a potato wart disease resistance gene Sen3
}

Charlotte Prodhomme, Danny Esselink, Theo Borm, Richard G. F. Visser, Herman J. van Eck and Jack H. Vossen*

\begin{abstract}
Background: Standard strategies to identify genomic regions involved in a specific trait variation are often limited by time and resource consuming genotyping methods. Other limiting pre-requisites are the phenotyping of large segregating populations or of diversity panels and the availability and quality of a closely related reference genome. To overcome these limitations, we designed efficient Comparative Subsequence Sets Analysis (CoSSA) workflows to identify haplotype specific SNPs linked to a trait of interest from Whole Genome Sequencing data.

Results: As a model, we used the resistance to Synchytrium endobioticum pathotypes 2, 6 and 18 that co-segregated in a tetraploid full sib population. Genomic DNA from both parents, pedigree genotypes, unrelated potato varieties lacking the wart resistance traits and pools of resistant and susceptible siblings were sequenced. Set algebra and depth filtering of subsequences ( $k$-mers) were used to delete unlinked and common SNPs and to enrich for SNPs from the haplotype(s) harboring the resistance gene(s). Using CoSSA, we identified a major and a minor effect locus. Upon comparison to the reference genome, it was inferred that the major resistance locus, referred to as Sen3, was located on the north arm of chromosome 11 between 1,259,552 and 1,519,485 bp. Furthermore, we could anchor the unanchored superscaffold DMB734 from the potato reference genome to a synthenous interval. CoSSA was also successful in identifying Sen 3 in a reference genome independent way thanks to the de novo assembly of paired end reads matching haplotype specific $k$-mers. The de novo assembly provided more $\mathrm{R}$ haplotype specific polymorphisms than the reference genome corresponding region. CoSSA also offers possibilities for pedigree analysis. The origin of Sen3 was traced back until Ora. Finally, the diagnostic power of the haplotype specific markers was shown using a panel of 56 tetraploid varieties.
\end{abstract}

Conclusions: COSSA is an efficient, robust and versatile set of workflows for the genetic analysis of a trait of interest using WGS data. Because the WGS data are used without intermediate reads mapping, CoSSA does not require the use of a reference genome. This approach allowed the identification of Sen3 and the design of haplotype specific, diagnostic markers.

Keywords: Bulked segregant analysis (BSA), Whole genome sequencing (WGS), Reference genome, $k$-mers, Diagnostic markers, Potato wart disease

*Correspondence: jack.vossen@wur.nl

Wageningen UR Plant Breeding, Droevendaalsesteeg 1,6708

PB Wageningen, The Netherlands 


\section{Background}

The identification of genetic loci involved in important traits variation is a fundamental step in genetic research and breeding. QTL mapping strategies are often timeconsuming and laborious. Often, large biparental populations $(n>300)$ segregating for the trait of interest are used, which necessitates considerable efforts for genotyping and phenotyping. Genome-Wide Association Studies (GWAS) are alternatives but equally require genotyping and phenotyping of a broad panel of individuals. Bulked Segregant Analysis (BSA) [1, 2], simplify the genotyping effort as pools of individuals with contrasting phenotypes are used, which drastically reduces the number of samples to be genotyped. BSA consists of estimating and comparing allelic frequencies between the pools. The frequency of non-linked loci for the trait of interest are expected to be equivalent between the pools whereas a bias in the frequency of the loci linked to the causal genes is expected. This way, DNA sequence variants linked to the trait of interest are identified, which allows the development of markers for genetic mapping and Marker Assisted Selection (MAS) purposes.

A conventional BSA approach uses a set of formerly identified markers [2,3] such as SNP-arrays [4]. Using markers not specifically developed for the population of interest can lead to ascertainment bias resulting in a limited number of markers, which may result in low mapping resolution. In polyploids, ascertainment bias is even a more severe problem, as markers in linkage disequilibrium are less informative. As sequencing became more affordable, genotyping-by-sequencing (GBS) methods, such as whole genome sequencing (WGS) [5], RNA-Seq [6] or different complexity reduction strategies [7, 8] have been applied in combination with BSA. These BSA methods, collectively referred to as mapping by sequencing (MBS) [9], involve the successive extraction, pooling and sequencing of the DNA of the pooled individuals. The reads obtained from these bulks are mapped to a reference genome and variants are called in each bulk. The allelic frequencies of the variants are compared between the bulks and variants in linkage disequilibrium with the locus encoding the trait of interest are identified. When WGS data are used, the resolution is increased and it becomes possible to not only identify polymorphisms in linkage disequilibrium but also in the causal locus itself [5].

Methods using read mapping rely on the availability of a reference genome. Nowadays, many crops have reference genomes available, but this is not yet the case for some non-model species or complex polyploid species. Even if a reference genome is available for the crop of interest, the assembly quality or completeness of the reference can be sub-optimal. Moreover, the genotype used as a reference can be considerably different from the genetic background of the population of interest. The wheat pan-genome was studied by comparing whole genome sequencing data of 18 different cultivars [10]. Interestingly, only $64 \%$ of the genes were present in all the cultivars and the rest showed presence-absence variations between them. This study illustrates why reference genome based approaches for genetic analysis and crop improvement can cause limited and incomplete results. This limitation is even more prominent in the case of disease resistance genes, which reside in clusters of highly similar paralogous sequences inherently difficult to assemble and highly variable in organization. Moreover, the genotype used as a reference is often susceptible to the disease and lacks the causal $R$ gene paralog segregating in a mapping population. This is exemplified by the case of the fine-mapping and candidate gene analysis of a powdery mildew resistance QTL in barley [11]. After an exome capture experiment was performed using homozygous recombinants, reads were mapped to the barley reference genome. Surprisingly, heterozygous sites were observed in genomic regions containing $R$ genes. This could be explained by assuming the reference genome is missing some paralogs of an $R$ gene cluster. The reads from the recombinant lines coming from these missing paralogs wrongly mapped to similar paralogs. In this case, a de novo assembly of the wrongly mapped reads was needed to identify the candidate genes.

Working directly with WGS reads without alignment to a reference offers several advantages. The use of subsequences ( $k$-mers of $k$ nucleotides) instead of the full reads allows to bypass sequencing errors in the reads and allows unambiguous comparisons between samples thanks to their invariable length. A notable example of the use of $k$-mers is provided in a study aiming to identify variants associated with human diseases [12]. Here, the authors identified several disease-associated sequences that were not present in the human reference genome. A similar approach was applied in a panel of wheat accessions [13]. The authors developed a new method, AgRenSeq, which combines resistance gene enrichment (RenSeq) and a $k$-mer based GWAS. They phenotyped an Aegilops tauschii panel with different stem rust races and intended to identify stem rust resistance $(S r)$ genes. AgRenSeq was successfully applied to identify and clone four functional $R$ genes independently from a reference genome in a diversity panel.

In this paper, we describe a set of workflows including a BSA approach using $k$-mers instead of variants. The Comparative Subsequence Sets Analysis (CoSSA) is suitable to quickly identify haplotype specific SNPs to develop diagnostic markers linked to traits of interest, with or without a reference genome. As an example, we 
used a tetraploid potato (Solanum tuberosum) population segregating for potato wart disease resistance.

Potato wart disease is caused by the obligate biotrophic soil-borne Synchytrium endobioticum Chytridiomycete fungus, which can result in dramatic yield losses. This fungus produces spores that can persist in the soil for more than 40 years [14]. In addition, no chemicals are available to control the pathogen. For these reasons, quarantine regulations are imposed [15], forbidding the cultivation of potatoes on infested fields. In addition, in protection zones around the infested fields, only resistant varieties may be grown. Therefore, resistant varieties are an essential key to manage the disease. The Sen 1 locus providing resistance to pathotype 1 isolates has been mapped previously on the north arm of chromosome 11 [16]. Most potato varieties of the European gene pool are resistant to pathotype 1 , but only a few are resistant to pathotypes 2, 6 and 18, all of which are occasionally detected in most European countries. Until recently, other QTLs giving resistance to various patterns of pathotypes have been mapped in diploid [17, $18]$ and tetraploid populations [19-21]. However, markers with diagnostic value were not found frequently. Only recently, a combined approach of linkage mapping, BSA and RNAseq data in a dihaploid potato population derived from the tetraploid variety Karolin allowed the identification of markers with diagnostic value linked to pathotypes 6 and 18 resistance on the north arm of chromosome 11 [22].

In this study, we applied the CoSSA workflows to map the Synchytrium endobioticum pathotype 2, 6 and 18 resistance from the variety Kuba, and refer to it as Sen3. CoSSA was implemented with and without the use of the potato reference genome and was successful in both cases. Sen 3 was fine-mapped and haplotype specific markers close to the resistance gene were developed and validated for Marker Assisted Selection. CoSSA also allowed us to improve the potato reference genome that is poorly assembled in the region of interest. Different aspects and further applications of CoSSA, such as pedigree analysis, are described in this paper and compared to earlier approaches. We found that Sen 3 descended from the variety Ora and was indistinguishable from the resistance in the variety Karolin [21, 22].

\section{Materials and methods}

\section{Plant material and wart disease resistance phenotyping}

The potato variety Kuba (resistant to the pathotypes 1, 2, 6 , and 18 [23]) was crossed to the susceptible variety Ludmilla (resistant to the pathotype 1 only [23]), resulting in a full-sib tetraploid population (K*L) of 328 clones segregating for the pathotypes 2, 6 and 18 resistance (abbreviated as P2, P6 and P18 respectively).
Progeny clones and both parents were phenotyped in spring 2016 for potato wart disease resistance with the Glynne-Lemmerzahl [24, 25] method. For each progeny clone, five tubers were tested for pathotypes 2, 6 and 18 resistance. The isolates used for inoculation were respectively JKI P2(G1)-2009, JKI P6(O1)-2009 and JKI P18(T1)-2009. Resistance levels were scored from 1 (highly resistant, early defense necrosis) to 5 (highly susceptible, formation of small to large warts). Mean scores were calculated for each progeny clone (Additional file 1). Chi square tests were performed to analyze the resistance segregation ratio to the three tested pathotypes in the population.

To compose the susceptible bulk (S-bulk), 17 progeny clones fully susceptible to P2(G1), P6(O1) and P18(T1) in 2016 were selected. To compose the resistant bulk (R-bulk), 17 progeny clones that were fully resistant to the three pathotypes in 2016 were selected and were rephenotyped (six tubers) in 2017. The three isolates and phenotyping method used were the same as the previous year. All the phenotypic assays were performed in the Laboratory of Quarantine Organisms, Department of Plant Pathology, IHAR-PIB, Poland.

\section{DNA extraction, pooling and sequencing}

Genomic DNA of the bulked progeny clones, the two parents, the mother (Bzura), the suspected great-greatgrand-father (BRA9089) of Kuba and of four susceptible varieties (Alegria, Desiree, Kuras and VR808) was extracted from freshly harvested leaves according to [26]. DNA concentration was assessed using a Qubit Fluorometer (Invitrogen). For the S-bulk and the R-bulk, $59 \mathrm{ng}$ of DNA of each individual was pooled. For all WGS experiments, $1 \mu \mathrm{g}$ of (pooled) genomic DNA was used for library preparation and sequenced on an Illumina HiSeq 2000 platform producing $151 \mathrm{bp}$ paired end (PE) reads (Hartwig Medical foundation, Amsterdam, The Netherlands).

Genomic DNA of the entire full-sib population $(n=328)$ and of a panel of 56 resistant and susceptible potato varieties was extracted from freshly harvested leaves using a modified CTAB-method [27]. These varieties were selected to embody the different wart resistance sources present in the European breeding germplasm. The DNA concentration was estimated using a NanoDrop ND-1000 spectrophotometer (Thermo Scientific) and adjusted to a concentration of 5-50 ng/ $\mu$ l. The DNA quantity was confirmed and the quality was assessed on ethidium bromide containing agarose gels.

\section{Comparative subsequence sets analysis workflows}

The CoSSA workflow used in this study is illustrated in Fig. 1 . All the scripts, the software and the code used in 


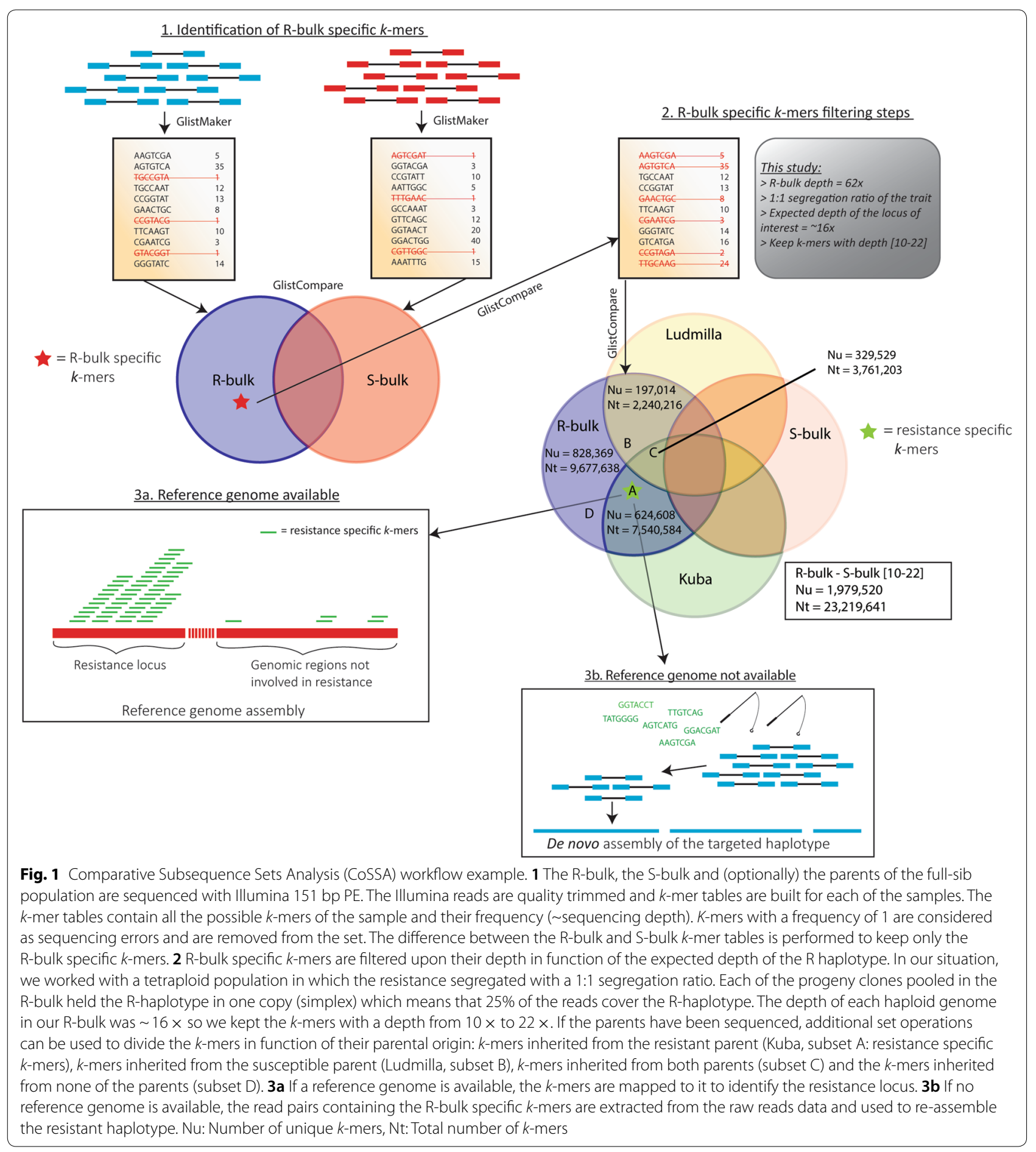

CoSSA are available on GitHub (https://github.com/ cprodhom/CoSSA-workflows). The computational requirements of each of the main steps of the workflows are given in Additional file 2. The forward and reverse reads were quality trimmed and Illumina adapters were removed using Trimmomatic version 0.32 [28] (settings
ILLUMINACLIP:TruSeq3-PE.fa:2:30:10, LEADING:3, TRAILING:3, SLIDINGWINDOW:4:15 and MINLEN:70). $K$-mer tables were built for each sequenced sample by using the GlistMaker program of the GenomeTester4 toolkit [29]. GlistMaker generates $k$-mers lists from the trimmed reads and counts the frequency 
$(\approx$ sequencing depth) of each unique $k$-mer. We used a $k$-mer size of 31 nucleotides. This $k$-mers size was chosen as a compromise between sequence uniqueness (increase the $k$-mer size increases the chances that each $k$-mer comes from a unique region of the genome) and sequence correctness (decrease the $k$-mer size allows to reduce the PE reads errors). The $k$-mers that were observed only once were removed from the dataset as they are likely caused by sequencing errors and would unnecessarily increase the required computational power and memory if retained. The GlistCompare program of GenomeTester4 was then used to perform basic set operations such as unions, intersections or differences.

To obtain $k$-mers exclusively present in the R-bulk (R-bulk specific $k$-mers) we subtracted the S-bulk $k$-mers from the R-bulk. Additional filtering steps were applied to refine the R-bulk specific $k$-mers selection. The sequencing yield was $52 \mathrm{~Gb}$ for the $\mathrm{R}$ bulk. Therefore, the sequencing depth of the R-bulk was approximately $62 \times$ considering a haploid genome of $840 \mathrm{Mb}$. Under the assumption of a uniform sequence coverage, the expected sequencing depth of the $\mathrm{R}$ haplotype is $\sim 16 \times$, as potato has a tetraploid genome $(2 \mathrm{n}=4 \times)$. Paralogous and other repetitive sequences are expected to be present at a multitude of this depth. In order to select for single copy SNPs specific for the resistant haplotype, we decided to keep $k$-mers with a depth from $10 \times$ to $22 \times$. These filtered R-bulk specific $k$-mers were further divided into four different sets after comparison with the $k$-mers from the resistant and susceptible parent: inherited from the resistant parent (referred hereafter as "resistance specific $k$-mers"), inherited from the susceptible parent, inherited from both parents or deriving from none of the parents. This last set, probably representing contamination with unrelated biological material, was discarded for subsequent analyses.

In order to select SNPs with high diagnostic value, prior to the development of PCR markers, $k$-mer sets operations were used to eliminate $k$-mers present in different varieties not sharing the same trait. $K$-mers tables were produced for Alegria, Desiree, Kuras and VR808 which are all resistant to the pathotype 1 but susceptible to P2, $\mathrm{P} 6$ and P18. The union of the $k$-mers from these varieties was subtracted from the resistance specific $k$-mers set.

For reference genome based downstream analyses, the resistance specific $k$-mers minus $\mathrm{S}$ varieties, the R-bulk specific $k$-mers inherited from the susceptible parent and the R-bulk specific $k$-mers inherited from both parents were mapped to identify the genomic region harboring the trait of interest. We used BWA aln (version 0.7.12) [30] to map the R-bulk specific $k$-mers to version v4.03 of the Solanum phureja DM1-3 (DM) genome [31, 32]. After mapping, $k$-mers per $1 \mathrm{Mb}$ bins, starting at the beginning of each chromosome, were counted using the bedtools suite (v2.25) [33]. The resulting numbers of $k$-mers per $1 \mathrm{Mb}$ bin were plotted using Microsoft Excel.

For reference genome independent downstream analysis, the paired end reads containing resistance specific $k$-mers were retrieved from the R-bulk and the resistant parent and, successively, assembled de novo. We used an in-house developed script to retrieve the PE reads containing at least one of the resistance specific $k$-mers. The de novo assembly of the extracted reads was done using SPADes (v3.11.1) [34] (command line options: -k 21, 33, 55, 77 -careful -pe1-1 -pe1-2 -pe2-1 -pe2-2). The corresponding scaffolds are mainly derived from the haplotype bearing the resistance and are referred to as the R-haplotype scaffolds. To compare the CoSSA workflows with and without using the reference genome, we mapped the resistance specific $k$-mers to the R-haplotype scaffolds using BWA aln and allowing no mismatches (command line option: $-\mathrm{n} 0$ ). The $k$-mers mapping to the three biggest R-haplotype scaffolds were extracted using SAMtools view (v1.3) [35] and mapped to the potato reference genome using BWA aln and allowing 0, 1 and 2 mismatches. The number of reads mapping to DM was determined using SAMtools flagstat.

$K$-mer sets algebra was also used for pedigree analyses. To check if different varieties sharing the same trait were identical by descent, the number of $k$-mers shared with the resistance specific $k$-mers were counted. For this purpose, the intersections between the resistance specific $k$-mers not present in the four susceptible varieties and the $k$-mers from the two pedigree clones (Bzura and BRA9089) were made. Successively, the resulting $k$-mers were mapped to the reference genome or to the R-haplotype scaffolds.

\section{Minimum input required by CoSSA}

To assess the influence of the sequencing depth on the CoSSA results, we randomly sampled subsets of reads from the R-bulk, the S-bulk, and the two parents using seqtk (v1.2) to simulate sequencing depths of $10 \times$ and $5 \times$ of the haploid genome. CoSSA was applied to these two datasets, changing only the R-bulk specific $k$-mers depth cut-off which was proportionally adapted to $k$-mers with a depth comprised between $6 \times$ and $14 \times$ and $k$-mers with a depth between $3 \times$ and $7 \times$ for the simulations with a depth of $10 \times$ and $5 \times$ respectively. To assess if we could identify the same loci with the lower depth datasets as with the full dataset, we calculated the signal to noise ratio (SNR) for the $10 \times$ and $5 \times$ simulations, with or without intersections from the parents. Without the parents intersections, the SNR was calculated as being the average number of R-bulk specific $k$-mers mapping under the peak (the peak boundaries are defined as 
identified with the full dataset) divided by the number of R-bulk specific $k$-mers mapping to the rest of the chromosome. If the parent's intersections were included, the SNR was calculated as being the ratio between the average of R-bulk specific $k$-mers coming from the specific donor parent under the peak and the average of R-bulk specific $k$-mers coming from the donor parent mapping to the rest of the chromosome.

To test if sequence input for the CoSSA workflows could be minimised further, we subtracted the S-bulk $k$-mers from the resistant parent (Kuba). We kept the $k$-mers from the difference with a depth comprised between $8 \times$ and $18 \times$ and mapped them to the reference genome. The number of mapped $k$-mers per $1 \mathrm{Mb}$ was counted and compared with the CoSSA results obtained with the two bulks and the two parents.

\section{Design of PCR markers for validation and diagnostic purposes}

A haplotype specific SNP without flanking SNPs within $30 \mathrm{bp}$ on each side has a maximum of $k(=31)$ resistance specific $k$-mers mapped to it. To select these "isolated haplotype specific SNPs" for the design of markers, we mapped the resistance specific $k$-mers minus the $S$ varieties $k$-mers to the potato reference genome DM (v4.03). To select SNPs coming from Ludmilla, we mapped the R-bulk specific $k$-mers inherited from Ludmilla to the reference genome. From the resulting .bam files, the position of the mapped $k$-mers was inferred using SAMtools and exported to Microsoft Excel. This way, we could pinpoint isolated haplotype specific SNPs because $31 k$-mers were mapped in a row and because there were no other $k$-mers mapped to the flanking $61 \mathrm{bp}$ sequences. The candidate SNPs were visualized in the bam file using the Integrative Genomics Viewer (IGV, v2.3.72) (Additional file 3).

To identify resistant haplotype specific SNPs without using the reference genome, we mapped the resistance specific $k$-mers minus the $S$ varieties $k$-mers to the de novo assembled R-haplotype scaffolds and isolated SNPs were pinpointed as described previously. As we mapped the resistance specific $k$-mers without the $S$ varieties $k$-mers to scaffolds belonging to the resistant haplotype, the susceptible variant(s) of the SNPs were not known. Knowing the susceptible variant(s) of the identified SNPs is necessary to design KASP markers. Therefore, we also mapped the R-bulk reads, which contain reads from the resistant haplotype and the seven possible susceptible haplotypes, to the de novo assembly using BWA mem (version 0.7.17). Duplicates were removed using sambamba (v0.5.1) [36]. The susceptible variant(s) of each selected SNP could be visualized in IGV.

\section{KASP markers genotyping}

A first set of Kompetitive Allele Specific Polymorphisms (KASP) markers (Additional file 4), that were used to validate the different CoSSA peaks, was designed by LGC Genomics (LGC, Hoddeston, UK) and tested in a subset $(n=83)$ of the $K^{*} L$ population. The clones from this subset were selected for historical reasons (first clones of the K*L population). The KASP markers name reflects the physical coordinate of the SNP, relative to DM. Allele specific forward primers and common reverse primers were designed based on the neighboring sequences of the $31 k$-mers from CoSSA mapped to the reference genome. One KASP marker (chr11_1259552) flanking the resistance locus and two new KASP markers designed for further fine-mapping (chr11_1519485 and chr11_1666090; Additional file 4) were used to genotype the full population $(\mathrm{n}=328)$. Two haplotype specific markers (chr11_1259552, chr11_1772869; Additional file 4) flanking the resistance locus were used to genotype an independent panel of 56 susceptible and resistant potato varieties. The KASP genotyping assays were performed by C. Meijer BV (Rilland, The Netherlands) according to the manufacturer's instructions (LGC Genomics). In brief, $1.5 \mu \mathrm{L}$ DNA of a concentration of $5-50 \mathrm{ng} / \mu \mathrm{L}$ in a 1536-well plate was dried in a fan oven for $1 \mathrm{~h}$ at $55^{\circ} \mathrm{C}$ and $1 \mu \mathrm{L}$ of the PCR mix was added (containing the $2 \times$ KASP Master mix, the primer mix and the water). The PCR was performed in a Hydrocycler 16 and the data reading was performed on a BMG PHERAstar ${ }^{\circledR}$

A second set of three KASP markers (NODE1_7193, NODE2_18410, NODE3_17594; Additional file 4) that were used to validate the "CoSSA without reference genome" workflow was designed in house using Primer3 [37]. The three markers were designed for SNPs from the three longest R-haplotype scaffolds and tested in the subset of $83 \mathrm{~K}$ "L progeny clones. These KASP assays were performed according to the manufacturer's guidelines (LGC Genomics, Hoddesdon, UK) in a total volume of $10 \mu \mathrm{L}$ with 5 to $50 \mathrm{ng}$ of genomic DNA on a BioRad CFX96 ${ }^{\mathrm{TM}}$ Real-Time System machine.

$\mathrm{Chi}^{2}$ tests were used to test the goodness of fit of the segregation of the tested markers with the expected 1:1 segregation ratio. Kruskal-Wallis tests were performed using R v3.2.3 to validate the association of the tested markers with wart disease resistance.

\section{Results}

\section{Distribution of resistance in the $K^{*} \mathrm{~L}$ population}

The resistance of the entire full-sib population $(n=328)$ and the two parents to pathotypes 2, 6 and 18 was assessed (Additional file 1). The distribution of resistance to the pathotypes 2 and 6 segregated in a bimodal fashion 
in a 1:1 ratio $\left(\chi^{2}\right.$ test $p$-value $>0.05$; Additional file $5 \mathrm{~A}, \mathrm{~B}$, E). The pathotype 18 scores were skewed towards susceptibility ( $x^{2}$ test $p$-value $<0.001$; Additional file $5 \mathrm{C}$ ) which can be explained by a weaker resistance of Kuba to pathotype 18. Nevertheless, the Pearson correlations between the resistance scores for the three pathotypes were very high (Additional file 5D): 0.93 between P2 and P6, 0.91 between P6 and P18 and 0.89 between P2 and P18. These observations suggested the presence of a single major resistance locus for all three pathotypes and possibly a minor effect locus improving resistance to pathotype 18 . When transforming the quantitative scores into a qualitative trait (Resistant or Susceptible, Additional file 1), we observed a perfect co-segregation between the three pathotypes. Only a few exceptions were found where the phenotypic classification for resistance against P2, P6 and P18 did not co-segregate. Most of these exceptions might be explained by the choice of the threshold to call a clone resistant or susceptible.

\section{Design of a COSSA workflows for gene mapping}

To identify sequence variants linked to the wart disease resistance from the $\mathrm{K}^{*} \mathrm{~L}$ population, we pursued a Bulked Segregant Analysis combined with Whole Genome Sequencing. We selected 17 fully resistant and 17 fully susceptible plants to the three pathotypes to compose a resistant bulk (R-Bulk) and a susceptible bulk (S-Bulk), respectively. Selected clones are highlighted in Additional file 1. The R-bulk, the S-bulk, Kuba (R parent) and Ludmilla (S parent) were sequenced with Illumina $151 \mathrm{bp}$ PE reads. The calculated sequencing depth obtained for each sample was of $62 \times, 82 \times, 72 \times$ and $70 \times$, respectively, assuming an $840 \mathrm{Mb}$ genome (Additional file 6). It was decided not to use complete reads but subsequences $(k$-mers) of 31 nucleotides instead. Indeed, sequences with an invariable length can be unambiguously compared using set algebra. These basic set operations are less computationally demanding than methods requiring reads alignment to a reference and allow to work independently from a reference. $K$-mer tables $(k=31)$, containing the unique $k$-mers and their frequency, for the four samples were produced and the $k$-mers with a depth of one were removed (Additional file 6). For Kuba and Ludmilla, we observed peaks of $k$-mers at four different $k$-mer frequencies. They corresponded to sequencing errors and PCR duplicates (low depth), the $k$-mers present in one haplotype (simplex; 13 for both Kuba and Ludmilla), two haplotypes (duplex), and the $k$-mers present in three haplotypes (triplex) or four haplotypes (quadruplex) (Additional file 7). The $k$-mers with a depth $>52(4 \times 13 \times)$ were assumed to be originating from multi-copy sequences. Except for the first two peaks (errors and single copy), no distinct frequency peaks could be observed in the bulks.
Most likely, the remaining peaks are diffuse because the bulk samples were composed of 17 different DNA samples from individuals containing genetic variants from eight haplotypes. From these eight haplotypes, only the resistant haplotype was present in each R-bulk individual which, theoretically, would be twice the frequency of the single copy peak ( $2 * 8=16$ for the R haplotype).

In order to select $k$-mers linked to resistance, we subtracted the S-bulk $k$-mers $(\mathrm{Nu}$ (number of unique $k$-mers) $=1,669,156,268$; $\mathrm{Nt}$ (total number of $k$-mers $)=45,467,875,801)$ from the R-bulk $k$-mers set $\quad(\mathrm{Nu}=1,601,951,563 ; \quad \mathrm{Nt}=34,475,435,516) \quad$ to obtain the R-bulk specific $k$-mers $(\mathrm{Nu}=88,774,024$; $\mathrm{Nt}=260,595,070$ ) (Additional file 6). After enriching for simplex $k$-mers using a $k$-mer depth threshold between $10<\mathrm{Nt} k$-mers $<23$, we retained $1,979,520$ unique $k$-mers $(\mathrm{Nt}=23,219,641)$. These unique $k$-mers were then divided into four different subgroups: 624,608 unique $k$-mers inherited from Kuba (resistance specific $k$-mers), 197,014 unique $k$-mers inherited from Ludmilla, 329,529 unique $k$-mers inherited from both parents and 828,369 unique $k$-mers inherited from none of the parents. From this last set of $k$-mers, $69 \%$ could be mapped to the potato reference genome. This set was not used for further analysis because they were likely due to sequencing errors and DNA contamination from an unrelated potato genotype.

To further increase the haplotype specificity of the resistance specific $k$-mers, we removed from the resistance specific $k$-mers the $k$-mers that were also found in the susceptible varieties Alegria, Desiree, Kuras and VR808 (S varieties). The sequencing depth obtained respectively for each tetraploid variety was $46 \times, 46 \times$, $43 \times$ and $45 \times$ (Additional file 6). From 624,608 resistance specific $k$-mers, $319,443 k$-mers remained after subtracting the $S$ varieties $k$-mers (Additional files 6 and 8 ). The unique resistance specific $k$-mers minus the $S$ varieties and the unique R-bulk specific $k$-mers inherited from Ludmilla and from both parents $(845,986$ unique $k$-mers in total) were mapped to $1 \mathrm{Mb}$ bins of the potato reference genome DM (Fig. 2, Additional file 8 ). The highest $k$-mers peak was identified on the first $5 \mathrm{Mb}$ of chromosome 11, where $13 \%$ of all the $\mathrm{R}$-bulk specific $k$-mers sets mapped. From these $k$-mers, $84.3 \%$ were inherited from Kuba, the resistant parent (resistance specific $k$-mers minus $\mathrm{S}$ varieties). Interestingly, we observed another peak of resistance specific $k$-mers mapping to the $20-21 \mathrm{Mb}$ bin of the chromosome 0 , and specifically to the unanchored superscaffold PGSC0003DMB000000734 (referred hereafter as DMB734). Several smaller peaks of $k$-mers could be observed in other genomic regions (Fig. 2). The larger and smaller $k$-mers peaks might represent major and 


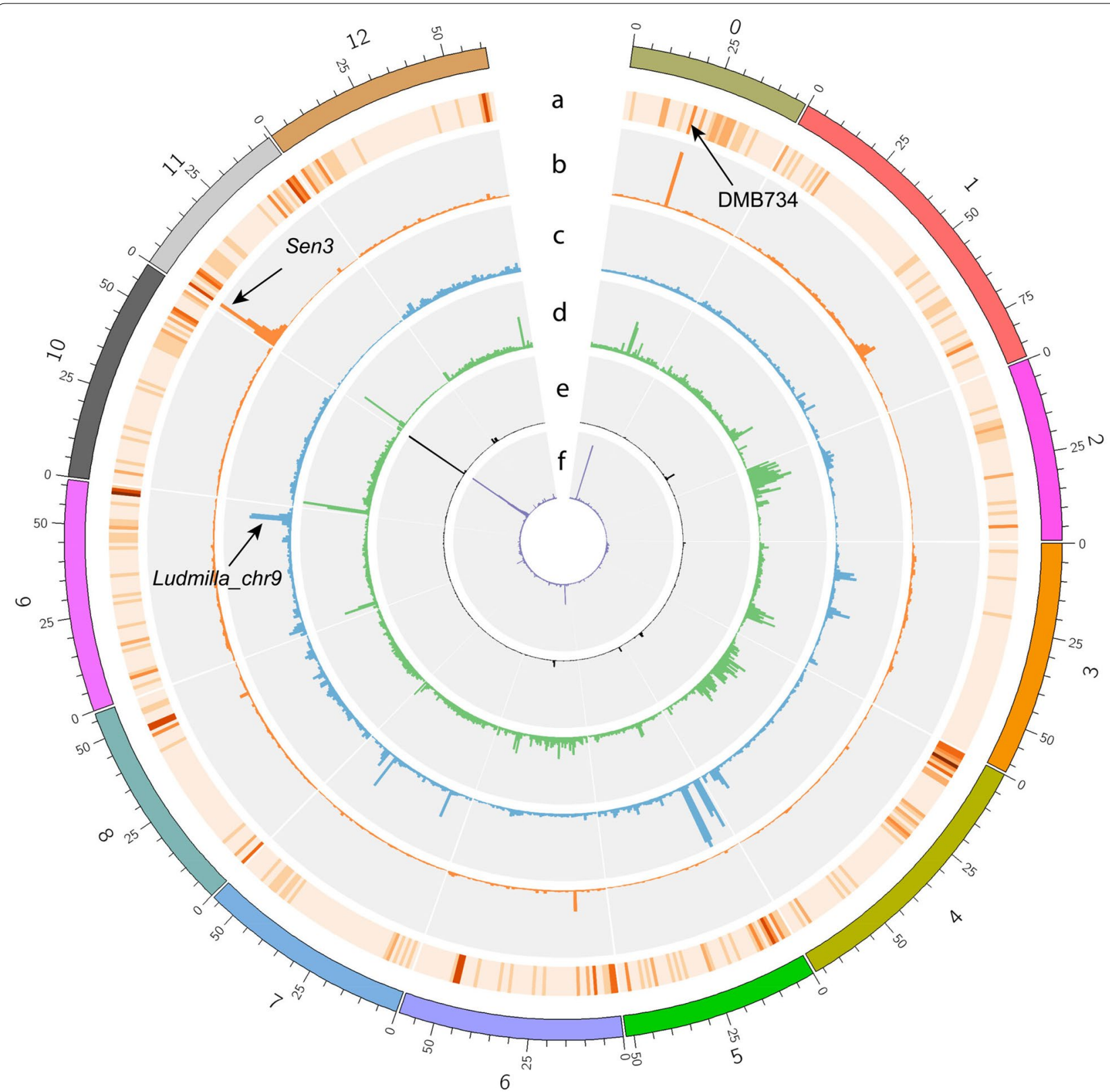

Fig. 2 Comparative subsequence sets analysis in the $K^{*} L$ population. The R-bulk specific $k$-mers from three different sets are mapped to the 12 chromosomes of DM and to the unanchored scaffolds (chromosome 0, v4.03). For each chromosome, the average of the number of R-bulk specific $k$-mers mapped has been calculated and for each $1 \mathrm{Mb}$ bin, the number of $k$-mers mapped from each set has been transformed to a percentage of the chromosome average. a Number of NLR genes per bin (1 Mb) from the DM genome according to [7]. b R-bulk specific $k$-mers minus $k$-mers present in the susceptible varieties inherited from Kuba $\left(y_{\max }=1208 \%\right)$, $\mathbf{c}$ the $k$-mers inherited from Ludmilla $\left(y_{\max }=469 \%\right)$, $\mathbf{d}$ the $k$-mers inherited from both parents $\left(y_{\max }=516 \%\right)$, e intersection of the $k$-mers from $\mathbf{b}$ with the $k$-mers from BRA9089 $\left(y_{\max }=428 \%\right)$, $\mathbf{f}$ intersection of the $k$-mers from b with the $k$-mers from Bzura $\left(y_{\max }=1205 \%\right)$. The arrows indicate the validated resistance loci and the position of the DMB734 scaffold

minor effect loci, a hypothesis that required validation. We decided to design markers to validate the two major peaks and five minor peaks of R-bulk specific $k$-mers coming from Ludmilla: on chromosome 3 (14$17 \mathrm{Mb} ; 0.39 \%$ of the mapped $3 \mathrm{R}$-bulk specific $k$-mers sets), chromosome 4 (64-68 Mb; 0.61\%), chromosome
5 (0-3 Mb; 1.46\%; 7-10 Mb; 2.1\%), and chromosome 9 (55-57 Mb; 0.62\%) (Additional file 8).

\section{Validation of the CoSSA results}

To validate the hypothesised resistance loci positions as identified with CoSSA, we set out to design haplotype 
Table 1 Validation of the CoSSA SNPs

\begin{tabular}{|c|c|c|c|c|c|c|}
\hline SNP & $\mathrm{R}$ allele donor & $\begin{array}{l}\text { Association with P2 } \\
\text { resistance* }\end{array}$ & $\begin{array}{l}\text { Association with P6 } \\
\text { resistance* }\end{array}$ & $\begin{array}{l}\text { Association with P18 } \\
\text { resistance* }\end{array}$ & $x^{2}$ & $\begin{array}{l}\mathrm{X}^{2} \text { test } \\
\text { significance }\end{array}$ \\
\hline chr00_20792298 & Kuba & $2.1 \mathrm{E}-14$ & $1.2 \mathrm{E}-14$ & $8.3 E-15$ & 1.5 & ns \\
\hline chr00_20801212 & Kuba & $1.3 \mathrm{E}-14$ & $7.3 \mathrm{E}-15$ & $4.8 \mathrm{E}-15$ & 1.2 & ns \\
\hline chr00_20858042 & Kuba & $2.1 \mathrm{E}-14$ & $1.2 \mathrm{E}-14$ & $8.3 E-15$ & 1.5 & ns \\
\hline chr00_20872536 & Kuba & $1.3 \mathrm{E}-14$ & $7.3 E-15$ & $4.8 \mathrm{E}-15$ & 1.2 & ns \\
\hline chr03_15683229 & Ludmilla & $6.8 \mathrm{E}-01$ & $6.9 \mathrm{E}-01$ & $3.0 \mathrm{E}-01$ & 0.1 & ns \\
\hline chr04_64488861 & Ludmilla & $6.6 \mathrm{E}-01$ & $9.7 \mathrm{E}-01$ & $3.8 \mathrm{E}-01$ & 0.1 & ns \\
\hline chr04_65204393 & Ludmilla & $4.5 \mathrm{E}-01$ & $1.1 \mathrm{E}-01$ & $2.0 \mathrm{E}-01$ & 1.5 & ns \\
\hline chr05_2117034 & Ludmilla & $3.8 \mathrm{E}-01$ & $9.4 \mathrm{E}-02$ & $1.4 \mathrm{E}-01$ & 0.0 & ns \\
\hline chr05_8795719 & Ludmilla & $2.0 \mathrm{E}-01$ & $9.0 \mathrm{E}-02$ & $1.6 \mathrm{E}-01$ & 0.0 & ns \\
\hline chr05_9254557 & Ludmilla & $2.3 \mathrm{E}-01$ & $8.4 \mathrm{E}-02$ & $1.8 \mathrm{E}-01$ & 0.0 & ns \\
\hline chr09_55113777 & Ludmilla & $2.4 \mathrm{E}-03$ & $9.0 \mathrm{E}-04$ & $4.2 \mathrm{E}-03$ & 0.0 & ns \\
\hline chr09_55354021 & Ludmilla & $4.6 \mathrm{E}-03$ & $1.3 \mathrm{E}-03$ & $5.9 \mathrm{E}-03$ & 0.1 & ns \\
\hline chr11_799292 & Kuba & $7.3 \mathrm{E}-13$ & $6.7 \mathrm{E}-14$ & $1.5 \mathrm{E}-13$ & 0.4 & ns \\
\hline chr11_1259552 & Kuba & $2.0 \mathrm{E}-12$ & $1.8 \mathrm{E}-13$ & $4.5 \mathrm{E}-13$ & 0.8 & ns \\
\hline chr11_1680269 & Kuba & $2.2 \mathrm{E}-14$ & $1.2 \mathrm{E}-14$ & 7.7E-15 & 1.5 & ns \\
\hline chr11_1772869 & Kuba & $2.9 E-13$ & $1.8 \mathrm{E}-13$ & $1.4 \mathrm{E}-13$ & 0.6 & ns \\
\hline chr11_1919971 & Kuba & $2.2 E-13$ & $1.4 \mathrm{E}-13$ & $1.1 \mathrm{E}-13$ & 0.8 & ns \\
\hline chr11_2040481 & Kuba & $2.2 \mathrm{E}-13$ & $1.4 \mathrm{E}-13$ & $1.1 \mathrm{E}-13$ & 0.8 & ns \\
\hline chr11_2298154 & Kuba & $2.1 \mathrm{E}-12$ & $9.7 E-12$ & $1.1 \mathrm{E}-12$ & 1.8 & ns \\
\hline chr11_2687396 & Kuba & $3.6 \mathrm{E}-11$ & $6.0 E-11$ & $1.9 \mathrm{E}-11$ & 2.1 & ns \\
\hline chr11_2815640 & Kuba & $2.5 \mathrm{E}-11$ & $5.5 \mathrm{E}-11$ & $1.4 \mathrm{E}-11$ & 1.5 & ns \\
\hline chr11_3325292 & Kuba & $1.2 \mathrm{E}-09$ & $4.6 \mathrm{E}-09$ & $7.7 \mathrm{E}-10$ & 2.1 & ns \\
\hline chr11_4362386 & Kuba & $1.0 \mathrm{E}-09$ & $3.0 \mathrm{E}-08$ & $3.4 \mathrm{E}-09$ & 1.0 & ns \\
\hline chr11_5505183 & Kuba & $4.8 \mathrm{E}-06$ & $6.4 \mathrm{E}-04$ & 4.3E-04 & 6.5 & $<0.01$ \\
\hline
\end{tabular}

$X^{2}$ tests: null hypothesis: the marker follows a 1:1 segregation pattern

ns not significant

${ }^{*} p$-values of association between KASP marker results and Glynne-Lemmerzahl tests of 2016 (Kruskal-Wallis tests)

specific KASP markers for the two larger and five of the smaller $k$-mers peaks identified. To increase the chances to select SNPs with diagnostic value, we removed from the resistance specific $k$-mers the $k$-mers that were also found in the susceptible varieties Alegria, Desiree, Kuras and VR808. When comparing the peak of resistance specific $k$-mers mapping to chromosome 11 before and after the subtraction of the $\mathrm{S}$ varieties $k$-mers (Additional file $8 \mathrm{~F}$ ), we observed that the highest peak shifted from the $3-4 \mathrm{Mb}$ bin to the $1-2 \mathrm{Mb}$ bin. This suggested that a high proportion of frequently occurring SNPs from multiple ancestral origin mapped to the 3-4 Mb bin.

From these $k$-mer sets, "isolated SNPs" were selected to design 24 KASP markers, covering all 7 CoSSA peaks, which were used to genotype a subset of 83 progeny clones from the $\mathrm{K}^{*} \mathrm{~L}$ population. $\mathrm{Chi}^{2}$ tests were used to validate the expected 1:1 segregation pattern of the markers (Table 1). All the markers followed the expected ratio except chr11_5505183 for which the segregation was skewed toward the alternative allele (Table 1; Additional file 1). The KASP markers from chromosome 11 and
DMB734 largely co-segregated and were strongly ( $p$-values $<0.001$ ) associated with pathotypes 2, 6 and 18 resistance as determined using Kruskal Wallis tests. Upon construction of a linkage map, it was found that DMB734 markers were positioned between markers corresponding to 1,259,552 bp and 1,772,869 bp of chromosome 11 . So most likely, DMB734 must be positioned inside one of the two gaps between DMB148 and DMB505. Despite this misassembly, the order of the chromosome 11 markers and the order of the markers on DMB734 were conserved within the Sen 3 locus in comparison with the reference genome.

Markers designed in four of the smaller CoSSA peaks were not associated with resistance ( $p$-values $>0.05$ ). Only the markers chr09_55113777 and chr09_55354021 were associated with pathotypes 2,6 and 18 resistance ( $p$-values $<0.01$; Table 1$)$. This second resistance locus was inherited from the susceptible parent Ludmilla and shows a smaller effect on resistance than the chromosome 11 locus (Additional file 9). This shows that CoSSA can identify major and minor loci, but also false positives. 


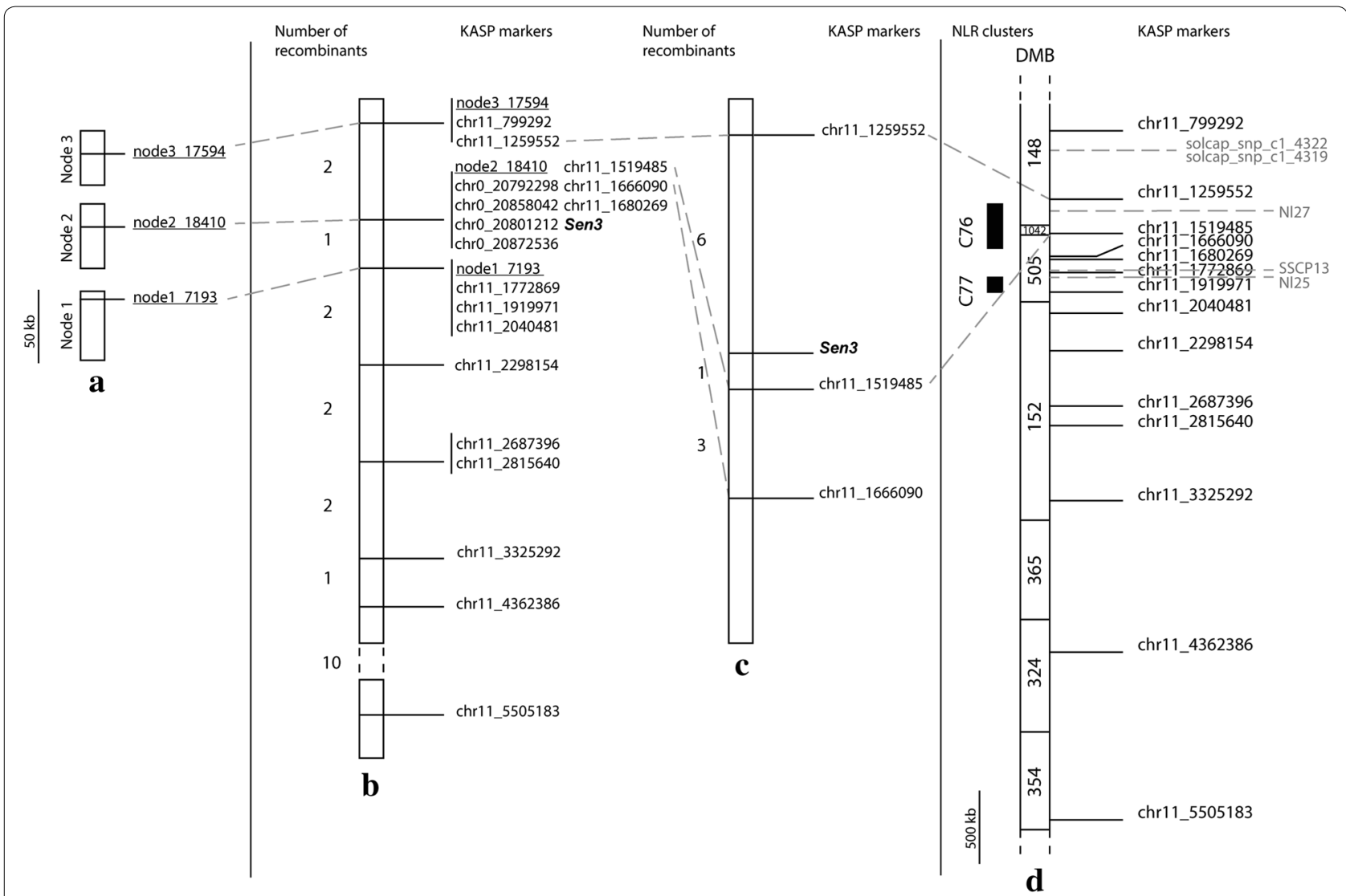

Fig. 3 Genetic and physical maps of the resistant haplotype. a Physical map of the three longest scaffolds of the R-haplotype de novo assembly. b Genetic map of the resistance region in a subset $(n=83)$ of the Kuba $\times$ Ludmilla population. The chromosome 0 scaffold could be anchored to the resistance interval. The analysis of recombinants allowed to fine-map the region flanked by chr11_1259552 and chr11_1772869. The markers developed without using the potato reference genome are underlined. c Genetic map of the resistance region in the full Kuba $\times$ Ludmilla population $(n=328)$. The resistance locus was fine-mapped to an interval between chr11_1259552 and chr11_1519485. $\mathbf{d}$ Physical map of the chromosome 11 first $6 \mathrm{Mb}$ region. The KASP markers developed to screen the Kuba x Ludmilla population are in black on the right of the haplotype and on the left are the NLR clusters [7]. The markers in grey are the markers used to map Sen 1 (NI25 and NI27) [16, 18] and to map Karolin's resistance (solcap markers and SSCP13) [22]

Hence, the SNPs identified in the peaks require validation through marker analysis.

\section{CoSSA data as a source for sequence variants in fine-mapping}

In the subset of 83 progeny clones, we could identify 20 recombinants between markers chr11_799292 and chr11_5505183 on the chromosome 11 haplotype (Fig. 3b; Additional file 1). Three informative recombinants indicated that the locus giving resistance to all the tested pathotypes, which we will refer to as Sen3, is within a 513,317 bp window relative to the reference genome (excluding DMB734; flanked by chr11_1259552 and chr11_1772869). We used the CoSSA resistance specific $k$-mers without the $S$ varieties $k$-mers to design two more KASP markers inside the resistance interval (chr11_1519485 and chr11_1666090). Screening of the full $K * \mathrm{~L}$ population $(\mathrm{n}=328)$ with chr11_1259552, chr11_1519485 and chr11_1666090 narrowed Sen3 to a $260 \mathrm{~kb}$ region between PGSC coordinates 1,259,552 bp and 1,519,485 bp (Fig. 3c). Interestingly, this genomic position contains many TNL (TIR Nucleotide-binding site Leucine-rich repeat) sequences (Fig. 3d) and matched the C76 [7] cluster which is synthenous to the XIa-TNL cluster [38] and RH11.1a clusters [39].

Only two false positives (K"L9006 and K*L9201) and one false negative (K"L9128) were observed in the full population $(n=328)$. Because of the scarcity of these observations, we can reasonably assume that they were due to mismatches between the samples used for the phenotyping and genotyping. None of the clones which showed a non-perfect co-segregation of the qualitative resistance traits to P2, P6 and P18 showed 
a recombination event between chr11_1259552 and chr11_1519485 (Additional file 1).

\section{CoSSA based gene mapping without a reference genome} Identification of sequence variants by mapping reads to a reference genome has its limitations. The genes or regions of interest may be absent or overly different from the reference. Especially $R$ gene clusters are difficult to assemble (as evident from the unanchored DMB734 contig; this study), and divergent, even among closely related haplotypes. We were interested to test if CoSSA could also be used without a reference genome. We extracted 408,281 and 469,251 read pairs from the R-bulk and from Kuba, respectively, which contained at least one of the resistance specific $k$-mers and assembled them. The obtained de novo assembly was composed of 21,306 scaffolds $\left(\mathrm{N}_{50}=1115\right)$ ranging in size from 100 until 46,690 bp (Additional file 10). To locate the R haplotype specific variants, we mapped the resistance specific $k$-mers minus the $S$ varieties $k$-mers to the de novo scaffolds. "Isolated SNPs" were selected to avoid KASP assay failure due to (unknown) flanking variants. To know the susceptible allele(s) of each DNA sequence variant, we also mapped the R-bulk reads to the de novo scaffolds. Next, we designed one KASP marker on each of the three longest scaffolds (node $1=46,690 \mathrm{~kb}$, node $2=44,756 \mathrm{~kb}$, and node $3=38,969 \mathrm{~kb}$; Fig. $3 \mathrm{a}$ ) and tested them in the subset of 83 offspring. The three markers were indeed linked to resistance and could be placed in the linkage map (Fig. 3b). The three markers were specific to the resistant haplotype and the markers present on node3 and node1 were flanking the resistance gene, while the node2 marker fully co-segregated.

To further inquire the benefit of the CoSSA workflow without a reference genome, we mapped the resistance specific $k$-mers to the three longest R-haplotype scaffolds (node1, node 2 and node3) allowing no mismatches. The mapped $k$-mers were extracted and mapped to the potato reference genome allowing 0,1 and 2 mismatches (Additional file 11). When no mismatches were allowed, only $2.95 \%, 0.58 \%$ and $41.85 \%$ of the $k$-mers mapping to the nodes 1,2 and 3 respectively could map to the reference genome. When 1 mismatch was allowed (the mismatch corresponds to the resistance specific SNP present in each $k$-mer), $25.6 \%, 33.11 \%$ and $66.63 \%$ of the $k$-mers mapping to nodes 1,2 and 3 respectively mapped to the reference genome. We released even more the mapping stringency by allowing 2 mismatches during the mapping, which allowed $k$-mers containing 2 SNPs to be mapped. With these settings, $45.17 \%, 60.78 \%$ and $81.85 \%$ of the $k$-mers mapping to nodes 1,2 and 3 mapped to the reference. Overall, the nodes 1 and 2 R-haplotype scaffolds contained only a small portion of $k$-mers that mapped to the reference genome, which can be explained by poor assembly of the reference genome or by huge differences between the DM haplotype compared to the Sen3 haplotype. The node3 scaffold contained more $k$-mers, which could map to DM. According to our genetic map (Fig. 3b), the DM assembly deviates strongly from the Sen 3 haplotype in the region between the flanking markers, which corresponds to the $R$ genes clusters. These results clearly show the advantage of working with a reference genome independent approach.

\section{CoSSA-based pedigree analysis}

To assess which clone in the pedigree of Kuba has been the donor of resistance (Additional file 12), we sequenced BRA9089 (suggested great, great grandparent of Kuba) and Bzura (grandparent of Kuba) and obtained sequencing depths of $51 \times$ and $47 \times$ respectively. We used the resistance specific $k$-mers without the $S$ varieties $k$-mers and made the intersection with Bzura and BRA9089 $k$-mers sets. Bzura and BRA9089 shared respectively $75.7 \%$ and $6.8 \%$ of the resistance specific $k$-mers without the $\mathrm{S}$ varieties $k$-mers. These shared $k$-mers were mapped to the reference genome (Fig. 2). Along the seven first bins of chromosome 11 (Additional file 13A), Bzura shared $99.44 \%$ of the $k$-mers with Kuba whereas BRA9089 shared only $12.9 \%$ of the $k$-mers with Kuba. These results showed that Bzura shared the full resistant haplotype with Kuba but it was not the case for BRA9089. In order to rule out that crossover events on both sides of the causal gene had resulted in lack of similarity in the $k$-mers lists of Kuba and BRA9089, we focussed on the fine-mapped interval $(1,259,552-$ $1,519,485$ bp). BRA9089 shared $1.7 \%$ of the Kuba resistance specific $k$-mers whereas Bzura hold $99.4 \%$ of them. In the de novo assembled R-haplotype scaffolds we described previously, on scaffolds node 2 and node1 containing the markers flanking Sen3, Bzura shared 99.9\% of the R-bulk specific $k$-mers with Kuba and BRA9089 $0.02 \%$ only (Additional file 13B). These observations strongly suggested that the BRA9089 clone we sequenced was not the donor of resistance. ${ }^{1}$

\section{Robustness of COSSA based gene mapping}

In the CoSSA workflows, we applied a depth cut-off on the R-bulk specific $k$-mers (10 to $22 \times$ ). We wanted to assess how these upper and lower depth cut-offs

\footnotetext{
${ }^{1}$ To validate that the clone BRA9089 that we sequenced was indeed not the resistance donor, we decided to re-phenotype it. The phenotyping results showed that our sample of BRA9089 was indeed susceptible to pathotypes 1, 2, 6 and 18 (mean scores of 4.4, 4.2, 5 and 4.8 respectively). We also genotyped BRA9089 with chr11_1259552 and chr11_1772869 which was negative to the tested markers (Table 2).
} 
affected the CoSSA peaks identification. If the parents were sequenced and no lower cut-off was applied on the R-bulk specific $k$-mers (2-22 $\times$; Additional file 14A), the signal-to-noise (SNR) ratio of Sen3 (chromosome 11: $0-5 \mathrm{Mb}$ ) was drastically reduced from 45.15 to 6.04 (Additional file 14D). The signal from the peak was still 6 times higher than the noise from the rest of the chromosome so the peak was still identifiable. In the case of the minor effect locus from Ludmilla on chromosome 9 (55-57 Mb), that we will refer as Ludmilla_chr9, however, the SNR was reduced from 10.04 to 1.75 which was too low to identify the peak. Therefore, the lower depth cut-off was not necessary to identify the major effect locus but necessary to identify the minor effect one. When no upper cut-off was applied (Additional file 14C), the SNR of both the major effect and the minor effect loci were unaffected: the SNR of Sen3 was of 45.56 instead of 45.15 when an upper cut-off was applied and the SNR of Ludmilla_chr9 was of 10.11 instead of 10.04 (Additional file 14D). Therefore, the upper cut-off threshold does not affect the detection of the peaks but impacts the selection against multi copy SNPs.

To determine the minimum input to supply for CoSSA to be efficient, we analysed the CoSSA results when the parents' sequences were not included in the workflow. When sequencing only the two bulks, the SNR of the Sen3 peak was halved (45.15 to 22.19; Additional file 14D) but it was still sufficient to clearly identify the peak. For the minor effect locus, however, the SNR was reduced from 10.04 to 1.88 when the parents were not sequenced which was too low to identify the peak. Therefore, sequencing only the two bulks was sufficient to identify the major effect locus but not to identify the minor effect one. When the parents were not sequenced, the $k$-mers lower depth cut-off became vital, as the SNR of Sen3 was reduced from 22.19 (10 to $22 \times$ ) to 1.38 (2 to $22 \times$ ) which made the peak not identifiable. Moreover, new peaks arose in other positions of the genome (Additional file 14A). These peaks were composed of $k$-mers that were not inherited from either of the parents, likely due to DNA contamination from unrelated biological material as discussed previously. This contamination would not have been removed if the parents had not been sequenced, making the lower depth cut-off the only way to discard it.

To further minimize the sequence data input for CoSSA, we reduced the sequencing depth of the samples (two bulks and parents) to a depth per haploid genome of $10 \times$ and $5 \times$ instead of $16 \times$ with the full dataset used previously (Additional file 15; Fig. 4). The SNR of Sen 3 was reduced from $22.2(16 \times)$ to $3.02(10 \times)$ and $1.3(5 \times)$ if the parental origin of the $k$-mers was not included in the CoSSA workflow, (Fig. 4a, d). Therefore, a minimum sequencing depth of $10 \times$ per haploid genome was sufficient to identify Sen 3 when no parents were sequenced. However, new peaks arose on chromosomes 2, 4 and 5, which were false positives, as they were not identified with a depth of $16 \times$ per haploid genome (Fig. 4a). For the minor effect locus on chromosome $9,16 \times$ per haploid genome was already not sufficient for its identification when no parents were sequenced. When the parental origin of the $k$-mers was included in the workflow, Sen 3 SNR was reduced from $45.3(16 \times)$ to $5.5(10 \times)$ and 2 $(5 \times)$ (Fig. 4 b, e). Therefore, even a sequencing depth of $5 \times$ per haploid genome was sufficient to identify the major locus, Sen3, if the parents were sequenced. However, as observed previously, new peaks arose on other chromosomes but the chromosome 11 peak was still the highest (Fig. 4b). Regarding the minor QTL (Ludmilla chr9), the SNR was reduced from $10(16 \times)$ to $4.2(10 \times)$ and $2.3(5 \times)$ for $10 \times$ and $5 \times($ Fig. $4 c, f)$. However, many new peaks arose for the $10 \times$ and $5 \times$ datasets (Fig. 4 c). The identification of this locus would then require the design of many more markers for each of these peaks to discard false positives.

Finally, to reduce the CoSSA input by sequencing fewer samples, we simulated the situation where only the resistant parent (Kuba) and the susceptible bulk were sequenced. The difference between Kuba's $k$-mers sets and the S-bulk's $k$-mers was made. The $k$-mers with a depth comprised between 8 and $18 \times$, according to the peak $(n=13)$ of simplex $k$-mers in Kuba (Additional file 7), were mapped to the reference genome (Additional file 16). With this reduced input, CoSSA was successful to identify Sen3, which had an SNR of 7.39. Obviously, the minor QTL Ludmilla_chr9 was not found because it is absent from Kuba. However, one smaller peak arose on chromosome $2(0-13 \mathrm{Mb})$ with an SNR of 4.28. This peak might be an additional minor effect locus. Again, a validation would be required to rule-out the fact that it is a false positive peak.

\section{Diagnostic value of the haplotype specific markers for breeding}

To assess the diagnostic value of the resistant haplotype specific KASP markers to detect Sen3 in breeding clones and varieties, we genotyped an independent panel of 56 tetraploid resistant and susceptible varieties with the flanking markers chr11_1259552 and chr11_1772869 (Table 2). The resistant allele of the markers was absent in the tested varieties susceptible to the pathotypes 2,6 and 18, including the BRA9089 clone which we showed to lack the Sen 3 haplotype using CoSSA. In 17 of the tested varieties, both flanking markers scored positive. From these varieties, 14 (including Bzura that contained Sen 3 as determined using CoSSA) are known to be fully 


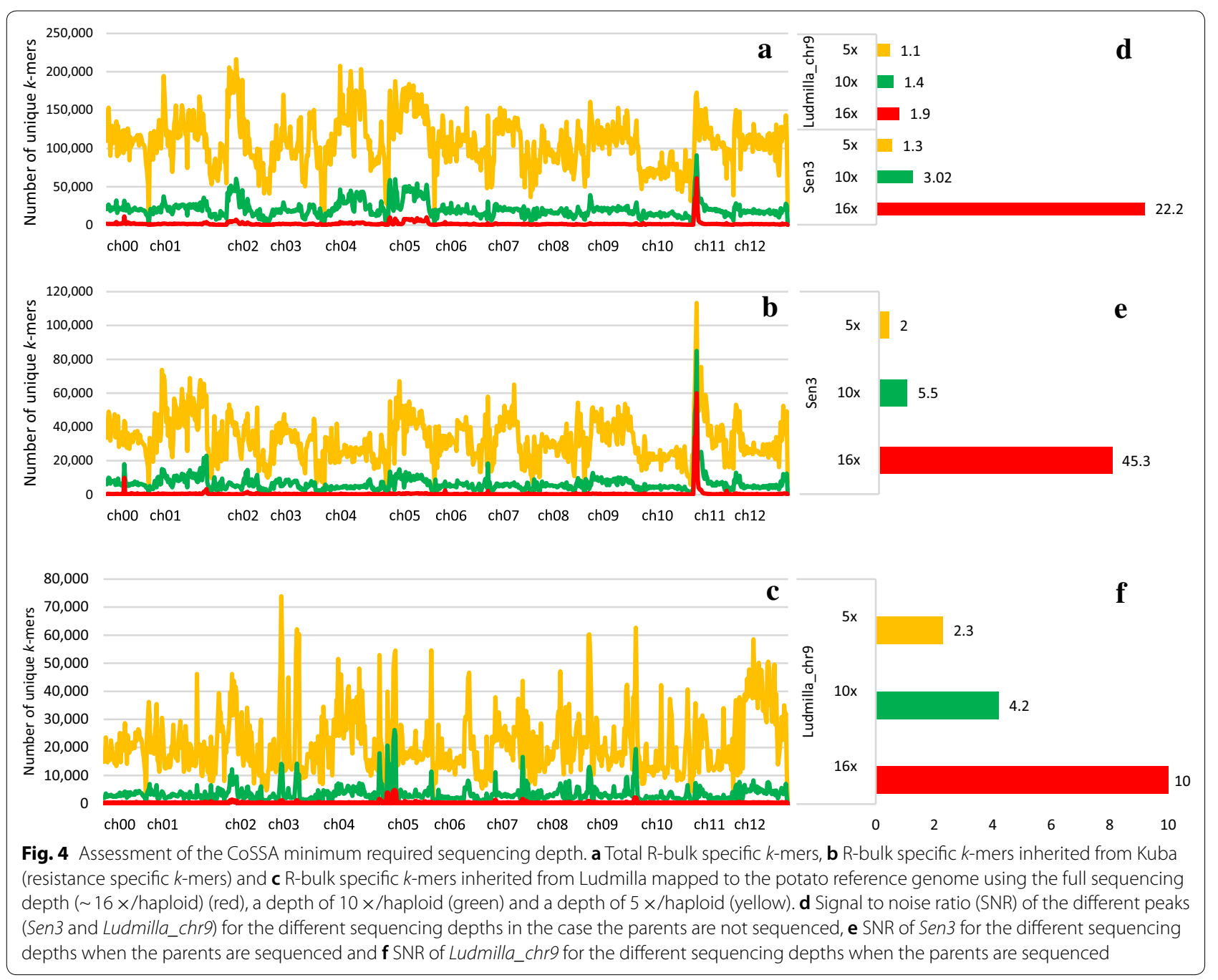

resistant to pathotypes 1, 2, 6 and 18. For the other three varieties (Gawin, Rudawa and Sonda), only a partial pathotype resistance pattern was known. We observed several false negative varieties in the dataset, which were resistant to the pathotypes 2, 6 and/or 18 but did not score positive to the tested markers. These varieties likely got their resistance from another source than Sen3.

\section{Discussion}

In this study, we designed CoSSA workflows for gene mapping and related applications in which sequence variants are deduced directly from subsequences ( $k$-mers), without the necessity of variant calling software. CoSSA is a quick and robust method to identify a locus involved in the variation of a particular trait. When the biggest datasets in this study were used, the jobs parallelized and run on only one node of $10.5 \mathrm{~Gb}$ memory, the CoSSA workflow using a reference genome took half a day. The reference independent workflow required around $30 \mathrm{~h}$.
The read pairs extraction step, currently consuming $23 \mathrm{~h}$ CPU, could be improved to be quicker (Additional file 2). In this case study, we mapped a major and a minor locus for resistance against pathotypes 2, 6 and 18 of Synchytrium endobioticum, the causal agent of potato wart disease. The CoSSA workflows also enabled to identify the major locus independently from a reference genome. Furthermore, CoSSA enabled to select for haplotype specific SNPs to fine-map the Sen3 locus corresponding to a $260 \mathrm{~kb}$ interval in the reference genome (between $1,259,552$ and 1,519,485 bp). The haplotype specific SNPs identified in this way proved to have diagnostic power in potato breeding material and pedigree analysis.

Synchytrium endobioticum pathotypes 2, 6 and 18 resistance had previously been identified in a similar region as the Sen3 locus as identified in this study [21, 22]. In the first study, the authors used 195 SSR markers to genotype the resistant parent Karolin and a susceptible parent which identity was not disclosed, as well as 
Table 2 Sen 3 markers validation in a panel of 56 varieties

\begin{tabular}{|c|c|c|c|c|c|c|c|c|c|c|c|}
\hline \multirow[t]{2}{*}{ Variety } & \multicolumn{2}{|c|}{ Registration } & \multicolumn{2}{|c|}{ Flanking makers } & \multicolumn{4}{|c|}{ Resistant to } & \multicolumn{3}{|c|}{ Clone in pedigree } \\
\hline & Country & Year & FM1 & FM2 & P1 & P2 & P6 & P18 & Ora & BRA-9089 & Capella \\
\hline Actaro & $\mathrm{HOL}$ & 2013 & 0 & 0 & $\mathrm{R}$ & $\mathrm{R}$ & $\mathrm{R}$ & $\mathrm{R}$ & & & \\
\hline Adam & $\mathrm{POL}$ & 2005 & 1 & 1 & $\mathrm{R}$ & $\mathrm{R}$ & $\mathrm{R}$ & $\mathrm{R}$ & $?$ & $?$ & $?$ \\
\hline Alegria & BRD & 2003 & 0 & 0 & $\mathrm{~S}$ & $\mathrm{~S}$ & $\mathrm{~S}$ & $\mathrm{~S}$ & & & \\
\hline AM78-3704 & $\mathrm{HOL}$ & & 0 & 0 & $\mathrm{R}$ & $\mathrm{R}$ & $\mathrm{R}$ & $\mathrm{R}$ & & & \\
\hline Andante & $\mathrm{BRD}$ & 2003 & 0 & 0 & $\mathrm{R}$ & $\mathrm{R}$ & $\mathrm{R}$ & $\mathrm{R}$ & & & \\
\hline Antares & DDR & 1961 & 0 & 0 & $\mathrm{R}$ & $\mathrm{R}$ & & $\mathrm{R}$ & & & \\
\hline Apollo & DDR & 1956 & 0 & 0 & $\mathrm{R}$ & $\mathrm{R}$ & & $\mathrm{R}$ & & & \\
\hline Aventra & $\mathrm{HOL}$ & 2005 & 0 & 0 & S & $\mathrm{R}$ & $\mathrm{R}$ & $\mathrm{R}$ & & & \\
\hline Axion & $\mathrm{HOL}$ & 2008 & 0 & 0 & $\mathrm{R}$ & $\mathrm{R}$ & $\mathrm{R}$ & $\mathrm{R}$ & & & \\
\hline Belita & $\mathrm{HOL}$ & 1986 & 0 & 0 & $\mathrm{R}$ & $\mathrm{R}$ & $\mathrm{R}$ & $\mathrm{R}$ & & & \\
\hline Bintje & $\mathrm{HOL}$ & 1910 & 0 & 0 & $\mathrm{~S}$ & S & $S$ & $S$ & & & \\
\hline BRA9089 & GER & & 0 & 0 & $S$ & $\mathrm{~S}$ & $S$ & $S$ & & & \\
\hline Bzura & $\mathrm{POL}$ & 1983 & 1 & 1 & $\mathrm{R}$ & $\mathrm{R}$ & $\mathrm{R}$ & $\mathrm{R}$ & y & y & $y$ \\
\hline Capella & GER & 1943 & 0 & 0 & & & & & & & \\
\hline Carrera & $\mathrm{HOL}$ & 1999 & 0 & 0 & $\mathrm{R}$ & $S$ & $\mathrm{R}$ & $S$ & & & \\
\hline Cekin & $\mathrm{POL}$ & 2004 & 1 & 1 & $\mathrm{R}$ & $\mathrm{R}$ & $\mathrm{R}$ & R & $?$ & $?$ & $?$ \\
\hline Celandine & $\mathrm{HOL}$ & 2013 & 0 & 0 & $\mathrm{~S}$ & $\mathrm{~S}$ & $\mathrm{R}$ & & & & \\
\hline Constantina & BRD & & 1 & 1 & $\mathrm{R}$ & R & $\mathrm{R}$ & $\mathrm{R}$ & $?$ & $?$ & $?$ \\
\hline Delcora & $\mathrm{HOL}$ & 1988 & 0 & 0 & $\mathrm{R}$ & $\mathrm{R}$ & $\mathrm{R}$ & $S$ & & & \\
\hline Deodara & GER & 1913 & 0 & 0 & S & $S$ & $S$ & $S$ & & & \\
\hline Desiree & $\mathrm{HOL}$ & 1962 & 0 & 0 & $\mathrm{R}$ & $S$ & $\mathrm{~S}$ & $S$ & & & \\
\hline Eurotonda & BRD & & 1 & 1 & $\mathrm{R}$ & $\mathrm{R}$ & $\mathrm{R}$ & $\mathrm{R}$ & $?$ & $?$ & $?$ \\
\hline Euroviva & BRD & & 0 & 0 & $\mathrm{R}$ & $\mathrm{R}$ & $\mathrm{R}$ & $\mathrm{R}$ & & & \\
\hline Gandawa & $\mathrm{POL}$ & 2004 & 1 & 1 & $\mathrm{R}$ & $\mathrm{R}$ & $\mathrm{R}$ & $\mathrm{R}$ & $?$ & $?$ & $?$ \\
\hline Gawin & $\mathrm{POL}$ & 2010 & 1 & 1 & $\mathrm{R}$ & & & $\mathrm{R}$ & $?$ & $?$ & $?$ \\
\hline Giewont & $\mathrm{POL}$ & 1955 & 0 & 0 & $\mathrm{R}$ & $S$ & $S$ & & & & \\
\hline Ibis & POL & 1987 & 1 & 1 & $\mathrm{R}$ & $\mathrm{R}$ & $\mathrm{R}$ & $\mathrm{R}$ & $n$ & y & y \\
\hline Ikar & $\mathrm{POL}$ & 1996 & 2 & 2 & $\mathrm{R}$ & $\mathrm{R}$ & $\mathrm{R}$ & $\mathrm{R}$ & $n$ & y & $y$ \\
\hline Karlena & DDR & 1988 & 0 & 0 & $\mathrm{R}$ & $S$ & $S$ & $S$ & & & \\
\hline Karolin & $\mathrm{BRD}$ & 1990 & 1 & 1 & $\mathrm{R}$ & $\mathrm{R}$ & $\mathrm{R}$ & $\mathrm{R}$ & y & y & y \\
\hline Kuba & $\mathrm{POL}$ & 1999 & 1 & 1 & $\mathrm{R}$ & $\mathrm{R}$ & $\mathrm{R}$ & $\mathrm{R}$ & y & y & y \\
\hline Kuras & $\mathrm{HOL}$ & 1996 & 0 & 0 & $\mathrm{R}$ & $\mathrm{S}$ & $\mathrm{S}$ & $\mathrm{S}$ & & & \\
\hline Lorch & SU & 1931 & 0 & 0 & S & & & & & & \\
\hline Ludmilla & BRD & 2008 & 0 & 0 & $\mathrm{R}$ & $S$ & $S$ & $S$ & & & \\
\hline Miriam & BRD & 1988 & 0 & 0 & $\mathrm{R}$ & $S$ & $\mathrm{~S}$ & $\mathrm{~S}$ & & & \\
\hline MPI44-1016-10 & GER & & 1 & 0 & $\mathrm{R}$ & $\mathrm{S}$ & $\mathrm{S}$ & S & $n$ & $n$ & $\mathrm{n}$ \\
\hline MPI44-1016-24 & GER & & 0 & 0 & $\mathrm{R}$ & $\mathrm{R}$ & $\mathrm{R}$ & $\mathrm{R}$ & & & \\
\hline Monalisa & $\mathrm{HOL}$ & 1982 & 0 & 0 & $\mathrm{R}$ & & & & & & \\
\hline Ora & DDR & 1952 & 1 & 1 & $\mathrm{R}$ & $\mathrm{R}$ & $\mathrm{R}$ & $\mathrm{R}$ & - & y & y \\
\hline Otolia & BRD & & 0 & 0 & $\mathrm{R}$ & $\mathrm{S}$ & $\mathrm{R}$ & $\mathrm{R}$ & & & \\
\hline Parella & $\mathrm{HOL}$ & & 0 & 0 & $\mathrm{R}$ & $\mathrm{R}$ & $\mathrm{R}$ & $S$ & & & \\
\hline Polessky Rosovy & SU & 1978 & 0 & 0 & & & & & & & \\
\hline Producent & $\mathrm{HOL}$ & 1984 & 0 & 0 & $\mathrm{R}$ & $\mathrm{s}$ & $\mathrm{S}$ & $\mathrm{S}$ & & & \\
\hline Rudawa & BRD & 2007 & 1 & 2 & $\mathrm{R}$ & $\mathrm{S}$ & $\mathrm{R}$ & $\mathrm{S}$ & $\mathrm{n}$ & y & y \\
\hline Saphir & $\mathrm{BRD}$ & 1960 & 0 & 0 & $\mathrm{R}$ & R & $\mathrm{R}$ & $\mathrm{R}$ & & & \\
\hline Smart & $\mathrm{HOL}$ & 2008 & 0 & 0 & $\mathrm{R}$ & S & $\mathrm{S}$ & $\mathrm{R}$ & & & \\
\hline Sonda & POL & & 1 & 1 & $\mathrm{R}$ & $\mathrm{R}$ & $S$ & $S$ & $?$ & $?$ & $?$ \\
\hline Spectra & $\mathrm{HOL}$ & 2016 & 0 & 0 & $\mathrm{R}$ & & $\mathrm{R}$ & & & & \\
\hline
\end{tabular}


Table 2 (continued)

\begin{tabular}{|c|c|c|c|c|c|c|c|c|c|c|c|}
\hline \multirow[t]{2}{*}{ Variety } & \multicolumn{2}{|c|}{ Registration } & \multicolumn{2}{|c|}{ Flanking makers } & \multicolumn{4}{|c|}{ Resistant to } & \multicolumn{3}{|c|}{ Clone in pedigree } \\
\hline & Country & Year & FM1 & FM2 & P1 & P2 & P6 & P18 & Ora & BRA-9089 & Capella \\
\hline Talent & $\mathrm{BRD}$ & 2006 & 0 & 0 & $\mathrm{R}$ & $R$ & $\mathrm{R}$ & S & & & \\
\hline Tivoli & DEN & 2002 & 0 & 0 & $\mathrm{R}$ & $\mathrm{R}$ & $\mathrm{R}$ & $\mathrm{R}$ & & & \\
\hline Tomensa & $\mathrm{BRD}$ & 1989 & 0 & 0 & $S$ & $S$ & $S$ & $S$ & & & \\
\hline Transit & $\mathrm{BRD}$ & 2009 & 1 & 2 & $\mathrm{R}$ & $\mathrm{R}$ & $\mathrm{R}$ & $\mathrm{R}$ & y & y & y \\
\hline Ulme & BRD & 1991 & 1 & 1 & $\mathrm{R}$ & $\mathrm{R}$ & $\mathrm{R}$ & $\mathrm{R}$ & y & y & y \\
\hline Ultra & $\mathrm{HOL}$ & 1999 & 0 & 0 & S & & $\mathrm{R}$ & & & & \\
\hline VR808 & $\mathrm{HOL}$ & 2009 & 0 & 0 & $\mathrm{R}$ & S & $S$ & S & & & \\
\hline Zagloba & GER & & 1 & 1 & $\mathrm{R}$ & $\mathrm{R}$ & $\mathrm{R}$ & $\mathrm{R}$ & y & y & y \\
\hline
\end{tabular}

Flanking markers of Sen3: FM1 = chr11_1259552 and FM2 = chr11_1772869. The dosage of the FM1 and FM2 is given (0: absent, 1: simplex, 2: duplex). Pedigree information is only shown if the variety was positive for the Sen 3 markers

?: No pedigree information is available, $\mathrm{n}$ : This clone is not in the pedigree, $\mathrm{y}$ : This clone is in the pedigree

two DNA bulks of a tetraploid offspring segregating for pathotypes 1, 2, 6 and 18 resistance [21]. Furthermore, the $8.3 \mathrm{k}$ SolCAP potato SNP array was used to identify markers significantly associated with resistance. The authors could not validate these markers with resistance in a broader panel of 91 distantly related varieties, probably because none of the markers was in coupling phase with the resistance gene. Such ascertainment bias can be circumvented only with haplotype specific SNPs. The failure to extrapolate SolCap markers associated with resistance from a biparental study to a GWAS panel should be expected because the SNP discovery panel of SolCap SNPs is not resistant to wart disease. In a later study, a dihaploid population was generated from the resistant variety Karolin and screened with the $12.8 \mathrm{k}$ SolCAP potato genotyping array [22]. The authors compensated the ascertainment bias caused by the SolCAP array by developing new haplotype specific markers using the RNAseq data obtained from three resistant descendants. The resistant locus was mapped to a region corresponding to a $777 \mathrm{~kb}$ interval of the reference genome (939,581 bp and 1,716,722 bp) which overlaps with the $260 \mathrm{~kb}$ interval we identified in this study.

In our study, we included Bzura, the resistant grandparent of the studied population, and BRA9089, a speculated donor for a resistance locus found in the German and Polish potato breeding material [40-42]. Among the 17 varieties from the tested panel holding the Sen3 markers, eight were bred in Germany and nine in Poland. Unfortunately, we could only trace back the resistant haplotype in Kuba's pedigree until Ora, a daughter of BRA9089 and Capella. Neither Capella, nor BRA9089 contained Sen3. We re-phenotyped the BRA9089 clone that we sequenced and it turned out to be susceptible to the four pathotypes. The impossibility to trace back further than Ora the haplotype in the pedigree could be due to pedigree errors. Another likely explanation is that the Capella and/or BRA9089 clones referred in literature are different from the clones we call Capella and BRA9089 today. Three out of the varieties that harbour the Sen3 markers do not contain Ora in their pedigree but do have Capella and BRA9089 (Table 2).

It is tempting to affirm that Kuba and Karolin share their resistance Identity-By-Descent. Unfortunately, insufficient pedigree information is available from Karolin. Kuba was tested with the flanking markers Kc8103 and RK36 and turned out to be positive [22]. We tested Karolin with chr11_1259552 and chr11_1772869 from the current study and it was positive as well. Moreover, the authors tested their markers in a panel of potato varieties, 15 of which were included in our panel as well. Gandawa, Gawin, Ibis, Ikar, Karolin, Kuba, Rudawa and Ulme are positive to our markers as well as the markers developed by [22]. However, the pedigree of these varieties offers many opportunities to conceive alternative routes of transmission of resistance genes from different pedigree branches. Our study enabled to trace back the Sen3 locus until Ora. However, Ora is not present in the pedigree of all of the above-mentioned positive varieties (Table 2). With the current information, we are not able to call the wart resistances from Karolin and Kuba identical by descent. CoSSA analysis would be suitable to prove sequence identity but genomic reads from Karolin are unfortunately not available. However, based on the markers produced in this study and on the S. endobioticum pathotype resistance pattern, the Kuba and Karolin resistances are indistinguishable.

Since its development almost 30 years ago [1, 2], Bulked Segregant Analysis strategies are used as an alternative to linkage map based QTL analysis and to identify new markers in unsaturated regions of genetic maps. BSA strategies have the main advantage of reducing 
the number of samples to be genotyped. In the case of CoSSA, we showed that the minimum requirement to identify Sen3 was to sequence two samples with contrasting phenotypes (R-bulk and S-bulk) with a sequencing depth for each pool of $10 \times$ per haploid genome (i.e. $40 \times$ for a tetraploid genome), reducing radically the genotyping costs. Indeed, we observed that even in the case of a relatively high number of $k$-mers which were not inherited from any of the parents (likely due to DNA contamination), applying a filter on the resistant bulk specific $k$-mers depth drastically improved the signal-tonoise ratio and allowed to identify the resistance locus segregating (Additional file 14A). If the two parents are included in the CoSSA workflow, the sequencing depth of the pools can be reduced further to $5 \times$ per haploid genome. Sequencing the two parents along with the bulks allowed to remove the previously described set of $k$-mers inherited from none of the parents. It has the other benefit of enabling the identification of minor effect loci. For instance, the minor effect locus inherited from Ludmilla on chromosome 9 would have not been identified without using the sequences of the two parents in the pipeline. Indeed, the SNR was too low, even with a sequencing depth of $16 \times$ per haploid genome, to be identified if the parents were not included in the analysis. Another alternative in CoSSA is to replace the resistant bulk by the resistant parent. This approach has the limitation that it will not identify minor QTLs from the susceptible parent. An advantage could be that it is more sensitive to minor QTLs from the resistant parent. A chromosome 2 peak $(0-13 \mathrm{Mb})$ was observed at the same position when using the full sequencing dataset (Fig. 2). This peak was ignored initially due to low SNR. In future research, we might be able to validate this hypothesis.

The set algebra applied in the current CoSSA workflow is made possible by the fixed length of the subsequences. Such an approach based on set algebra has been implemented in the NIKS algorithm [43] to identify the causal mutations in populations derived from EMS mutagenized plants. The beginning of the NIKS workflow is similar to CoSSA: pools of mutants and wild types are sequenced, $k$-mer lists are produced for each bulk and $k$-mers with a low frequency are removed. Subsequent steps in the CoSSA workflow are clearly different and serve different goals. NIKS workflow is designed to identify the mutation causal for a specific phenotype that segregates. Therefore, the NIKS workflow is a comparative subsequence sets analysis that identifies a "needle in the k-stack". The CoSSA workflows presented in our study are designed to identify haplotype specific "k-packs from the k-stack". Moreover, NIKS workflow is not suited to identify k-stacks linked to dominant genes like Sen 3 because NIKS requires the haplotype of interest to be homozygous. Therefore, comparative subsequence set analysis is a powerful approach to address different genetic questions, but each question will require a different workflow.

Although the set algebra allows quick and unambiguous comparisons between samples, it may be stringent as it discards all the first bulk $k$-mers that occur at least 2 times in the second bulk. Therefore, CoSSA works best for major QTLs that can be phenotyped unambiguously. If the phenotyping is not straightforward, linked $k$-mers will be present both in the susceptible and resistant bulks. The use of the set algebra might also have the consequence of missing minor effect loci that will be present in both bulks. A way to reduce the stringency is to increase the number of individuals that compose the bulks. CoSSA offers another way to reduce stringency by increasing the S-bulk $k$-mers depth cut-off, which would not require a bigger population. Instead of removing from the R-bulk all the $k$-mers that occur at least 2 times in the S-bulk, we could remove only the $k$-mers which occur more than $\frac{x}{n} \times m$ with $x$ the depth of the S-bulk, $n$ the number of individuals in the S-bulk, and $m$ the number of phenotyping mistakes allowed $(>0)$.

The selection of haplotype specific variants was improved by including four potato varieties susceptible to the wart disease. Including these varieties in the CoSSA workflow halved the number of resistance specific $k$-mers. In other words, without this extra-step, the markers we designed had only $50 \%$ of chance to be haplotype specific. Moreover, we showed that the peak shifted from 3-4 Mb to 1-2 Mb when removing common $k$-mers present in the four susceptible varieties. This suggested that the 1-2 Mb region contained SNPs with a higher haplotype specificity than the $3-4 \mathrm{Mb}$ region. This might be due to a recombination of the original Sen3 haplotype, which replaced the centromeric part with a more common haplotype. Therefore, after the removal of the susceptible varieties $k$-mers, only the $k$-mers derived from the Sen 3 introgression remained. We improved the SNPs selection as well by applying a depth cut-off on the resistance specific $k$-mers. The lower depth cut-off allowed to remove noise coming from $k$-mers that were not linked to resistance but were, by chance, not present in the S-bulk or noise coming from PCR duplicated reads containing sequencing errors. The upper cut-off, on the other hand, allowed to select for SNPs coming from single copy regions. The thresholds can be chosen by estimating the depth of the resistant haplotype according to the sequencing depth of the R-bulk, or by determining the $k$-mers frequency peaks such as in Additional file 7.

The markers specific to the Sen 3 haplotype were tested in an independent panel of 56 tetraploid varieties (Table 2) and their diagnostic value was tested. Indeed, 
none of the susceptible varieties tested were positive to the two flanking markers. Among the 17 varieties that were positive, 14 are known to be resistant to pathotypes 1, 2, 6 and 18. Gawin, Rudawa and Sonda are only known to be resistant to P1 in addition to P18, P6 and P2 respectively. These incomplete resistance patterns could be explained by the fact that: 1 . not all pathotypes were tested, 2. the isolates of the tested pathotypes used for phenotyping were derived from different sources and that their different genetic composition causes they are not recognised by Sen3, or 3. thresholds for resistance and susceptibility were not sufficiently uniform. We found in our $\mathrm{K}^{*} \mathrm{~L}$ population plants that harboured both flanking markers but that were only partially resistant to P18. Future analyses will learn what the genetic basis for the partial or complete P18 resistance conferred by Sen 3 is.

The previously discussed strategy to include more varieties without the trait of interest in the CoSSA workflow to remove common SNPs could be expanded to an association mapping type of approach. Instead of applying the method on a segregating population, CoSSA could as well be applied on bulks from a broader panel of genotypes with opposite phenotypes. This strategy would be successful only when the causal genes are identical by descent. A similar method, called AgRenSeq, has recently been developed [13] and proved to be efficient to map $R$ genes in Aegilops tauschii. The authors performed RenSeq, which requires prior knowledge about Nucleotide binding Leucine rich Repeat (NLR) genes repertoire and also introduces a bias to NLR rich regions of the genome and prevent the identification of other genes (non-NLR). CoSSA with WGS data is an unbiased genome wide approach that involves moderate sequencing costs and computation extensive $k$-mer counting instead of computation intensive reference genome dependent variant calling. An untargeted association mapping method based on the use of $k$-mers (HAWK) has been developed [12]. CoSSA differs from HAWK by its simplicity as it does not require advanced statistical methods or strong bioinformatics skills. Moreover, the last step of the CoSSA workflow consisting of assembling the read pairs containing the resistance specific $k$-mers instead of the $k$-mers themselves as in the HAWK pipeline should give a better quality of the assembly. Indeed, by using the $k$-mers, one loses the advantage of the paired end reads that can help resolving repetitive regions as well as duplicated or regions harbouring low complexity levels. We overcame this drawback by selecting the haplotype specific read pairs using the resistance specific $k$-mers.

The de novo assembly of the reads containing the resistance specific $k$-mers and the mapping of the resistance specific $k$-mers to the de novo scaffolds allows to reduce the number of candidate SNPs for which to design markers. Indeed, mapping-free SNP calling methods have been developed [44-46] and could be used in a BSA setup. However, without an assembly, more SNPs would have to be tested to map the locus. Moreover, having the sequences flanking the candidate variants improves the quality of the markers. Additionally, the de novo assemblies allow identification of candidate genes. For instance, [13] using NLR-parser [47] could search for NLR genes in de novo assemblies. However, in the case of NLR genes, it has been shown that a de novo assembly based on short reads might result in chimeric scaffolds [48]. In the same publication, by using PacBio long reads, the authors successfully assembled a complex resistance gene cluster and cloned the causal resistance gene.

In this paper, we mapped Sen3 to a region containing the complex $R$ gene cluster C76 which harbours 8 TNLs [7]. The first locus involved in potato wart disease resistance identified, Sen1, was also mapped to chromosome 11 near the C76 cluster [16] (Fig. 3). Sen3 could be a different paralog from the same cluster as Sen1, but it could also be an allelic variant of Sen1. Only fine-mapping and the cloning of these genes will answer this question. The C76 cluster is in fact even more complex as the assembly of the DM genome is incomplete in this region (Fig. 3). By adding contig DMB734, we added 3 TNLs and still at least one additional gap remains. This complexity was also reflected in the de novo assembly we generated from the resistant haplotype specific reads which was very scattered. Thus, we were unsuccessful in assembling the $R$ gene cluster using the short reads. However, our analyses showed clearly that our assembly of the R haplotype was more complete than DM. Only $11.9 \%$ of the resistance specific $k$-mers that mapped to the three longest scaffolds of the de novo assembly could be mapped back to the reference genome with a perfect match and only $38.2 \%$ could be mapped allowing one mismatch. This matched our observations that the chromosome $11 R$ gene cluster but also many other $R$ gene regions in DM are incomplete.

\section{Conclusions}

Overall, the CoSSA workflows were successful in mapping the potato wart disease resistance locus segregating in a full sib population of tetraploid non-inbred parents, with or without the use of the potato reference genome. This method is both quick and cheap as the bioinformatics pipeline required approximately only half a day and the price of whole genome sequencing using short reads is still decreasing. CoSSA is highly efficient as well: in the reference-free experiment, we needed to screen only three KASP markers to identify the resistance locus 
and design two valuable flanking markers. Moreover, we showed that the CoSSA de novo assembly of the $\mathrm{R}$ haplotype was more complete than the potato reference genome. The CoSSA workflows offer many possibilities to the user such as the quick mapping of major and minor effect loci, the identification of haplotype specific variants, the de novo assembly of haplotype specific reads and the in silico inspection of genotypes sharing the same trait. Finally, the different filters settings along the pipeline allow the user to adapt CoSSA to diverse crops and traits.

\section{Additional files}

Additional file 1. Phenotypic and genotypic data of the $K^{*} L$ population. The Excel sheet comprises: the genotypic data for all the tested markers, the number of tubers tested per genotype, the average score of the Glynne-Lemmerzahl assays performed in 2016 and 2017 for the pathotypes 2, 6 and 18, the individuals included in the subset $(n=83)$ used for the validation of the COSSA results and the individuals selected for the R-bulk and the S-bulk.

Additional file 2. CoSSA computational requirements. Computational requirements for the biggest datasets we used in the CoSSA workflows (sequencing depth of $16 \times$ /haploid genome). We showed that a depth of $10 \times /$ haploid genome is sufficient when the parents are not sequenced and $5 \times$ /haploid genome when parents are sequenced which reduces these computational requirements. The different jobs were executed on the WUR Plant Breeding server. Only one node of 10.5 GB memory was used. When the jobs are parallelized for the different COSSA samples, the reference genome dependent workflow can be run in half a day. For the reference genome independent CoSSA workflow, we advise to allocate more nodes to the reads extraction and to the de novo assembly steps as they require more time. The most time consuming step at the moment which could be improved is the script used to extract the read pairs containing the $k$-mers.

Additional file 3. Identification of isolated haplotype specific SNPs for markers development. To identify a good candidate SNP to design diagnostic markers, we mapped the resistance specific $k$-mers minus $S$ varieties to the reference genome and counted how many $k$-mers map to each chromosome bin. Each resistant haplotype specific SNP can have maximum $k$ k-mers (in our case, 31 31-mers) mapped to itself. We designed most KASP markers for SNPs which had 31 -mers mapped under the main $k$-mers peaks. Fluorescent dyes: $F=F A M ; H=H E X$.

Additional file 4. KASP primers used in the study. For each KASP marker used in the study is given: the marker name (chromosome_position), the experiment in which it was used, the population in which it was used, the two forward primer sequences and the common primer sequence.

Additional file 5 . Resistance distribution in the $K^{*} L$ population. Resistance distribution in the full-sib population $(n=328)$ for pathotypes 2 (A), 6 (B) and 18 (C). The phenotyping was performed with the Glynne-Lemmerzahl method in 2016. For each resistance category, the proportion of individuals with the R allele of Sen3 (black), without the R allele (grey) and the proportion of recombinants (black stripes) is given. In (D) are given the Pearson correlations between the resistance mean scores of P2, P6 and $\mathrm{P} 18$. Chi square tests were conducted to validate if the resistance to the three pathotypes segregated in a 1:1 ratio in the population (E).

Additional file 6. Summary of the sequencing data used and of the COSSA $k$-mers subsets. For all the samples sequenced, the number of reads and the sequencing depth is given. The number of unique $k$-mers $\mathrm{Nu}$ and the number of total $k$-mers $(\mathrm{Nt})$ is given for all the sequenced samples and several subsets obtained after basic set operations for different depth cut-offs.

Additional file 7. Distribution of the number of total $k$-mers in function of the $k$-mer depth. The total number of $k$-mers (unique $k$-mers $x$ depth) for each sequencing depth from 2 to 100 are represented in this graph for the 4 different samples (red: Kuba, blue: Ludmilla, green: R-bulk, yellow: S-bulk). For the two varieties samples (Kuba and Ludmilla), the peaks of k-mers from simplex, duplex, triplex and quadruplex regions are visible. There is a shift in the simplex peak of the bulks as there are 8 possible haplotypes instead of 4 as in the parental genotypes.

Additional file 8. CoSSA results with the reference genome. Number of R-bulk specific $k$-mers (depth 10 to 22) mapping to each $1 \mathrm{Mb}$ bin of (A) chromosome 0, (B) chromosome 3, (C) chromosome 4, (D) chromosome 5, (E) chromosome 9 and (F) chromosome 11 of the potato reference genome DM. Red: $k$-mers inherited from Kuba (resistance specific $k$-mers), yellow: $k$-mers inherited from Kuba minus the $k$-mers present in the susceptible varieties, blue: $k$-mers inherited from Ludmilla, green: $k$-mers inherited from both parents.

Additional file 9. Markers effects on pathotypes 2,6 and 18 resistance. Boxplots of the resistance scores for P2, P6 and P18 in function of the allele (0: S allele, 1: $R$ allele) of the KASP markers chr11_1519485 (A) and chr09_55113777 (B) in the Kuba x Ludmilla population subset $(n=83)$.

Additional file 10. De novo assembly statistics. Statistics of the de novo assembly of the resistant haplotype performed.

Additional file 11. Comparison of the COSSA workflows with/without reference. Number and percentage of the $k$-mers mapping to the three biggest scaffolds of the de novo assembly with 0 mismatches that map to the reference genome (DM) as well. During the mapping process to the reference, 0,1 and 2 mismatches were allowed.

Additional file 12. Pedigree of Kuba. The pedigree tree of Kuba (according to [49]) and the known resistance pattern of its ancestors. BRA9089 is present in the pedigree of several varieties resistant to pathotypes 2, 6 and 18 and is thought to be the ancestral donor of their resistance. BRA9089 is present in the resistant grand-parent pedigree (Bzura) and in the susceptible grand-parent (Karlena) pedigree as well.

Additional file 13. Pedigree CoSSA analysis. Number of unique $k$-mers in common between the resistance specific $k$-mers without the $S$ varieties $k$-mers and Bzura (the resistant parent of Kuba), BRA9089 (putative resistant donor) and $\mathrm{Kuba}$, (A) mapped to the reference genome (chromosome $11,0-7 \mathrm{Mb}, \mathrm{DMB} 734 \mathrm{k}$-mers were added to the $1-2 \mathrm{Mb}$ bin) (B) mapped to the 10 longest scaffolds of the de novo assembly. Orange: Kuba, blue: Bzura, black: BRA9089. The bars numbers represent the percentages of the intersection $k$-mers.

Additional file 14. Assessment of the depth cut-offs on the CoSSA results, Assessment of the effect of the lower and upper depth cut-off on the CoSSA output. (A) CoSSA output without the lower depth cut-off (R-bulk specific $k$-mers with a depth from 2 to $22 \times$ ), (B) CoSSA output with the lower and upper cut-offs (R-bulk specific $k$-mers with a depth from 10 to $22 \times$ ), (C) CoSSA output without the upper depth cut-off (R-bulk specific $k$-mers with a depth from 10 to $\infty$ ). Red: $k$-mers inherited from Kuba (resistance specific $k$-mers), blue: $k$-mers inherited from Ludmilla, green: $k$-mers inherited from both parents, grey: $k$-mers inherited from none of the parents. (D) Signal to noise ratio (SNR) for Sen3 and Ludmilla_chr9. Black: SNR for CoSSA results when no lower cut-off is applied (2 to $22 x$ ), stripped: SNR for CoSSA results when an upper and a lower cut-offs are applied (10 to $22 \times$ ), grey: SNR for CoSSA results when no upper cut-off is applied (22 to $\infty$ )

Additional file 15. Description of the datasets used in the COSSA simulations with lower sequencing depths. Sequencing depth of the full sequencing data ( $16 \times$ /haploid genome) and the two randomly sampled subsets to simulate depths of $10 \times$ and $5 \times$ per haploid genome. For each subset, the $k$-mers depth cut-off used in the CoSSA is given.

Additional file 16. CoSSA applied with Kuba and the S-bulk only. Kuba specific $k$-mers (depth $8 \times$ to $18 \times$ ) obtained by the difference between Kuba and the S-bulk mapped to the reference genome. 


\section{Acknowledgements}

We thank all the partners involved in this public-private partnership (Averis seeds BV, Böhm-Nordkartoffel Agrarproduktion GmbH \& Co. OHG, Danespo, HLB BV, HZPC Holland BV, C Meijer BV, SaKa Pflanzenzucht GmbH \& Co. KG and Teagasc) for providing invaluable potato material, phenotypic data, KASP marker analysis, and scientific discussions. Wilbert van Workum and Limes Innovations are acknowledged for facilitating WGS at Hartwig Medical. We acknowledge the very kind help from Dr. Danuta Sekrecka, Laboratory of Potato Gene Resources and Tissue Culture, IHAR, Department of Potato Protection and Seed Science at Bonin, Poland, for providing varieties. A warm thank you goes also to Jarosław Przetakiewicz and IHAR who phenotyped the $K^{*} L$ population. Klaus Dehmer from IPK Gross Lüsewitz Potato Collection is acknowledged for providing material of Capella and BRA9089. We finally acknowledge Bernd Truberg and Martin Sloksnat for inspiring discussions and pedigree information.

\section{Authors' contributions}

$\mathrm{CP}, \mathrm{HJvE}$ and JHV conceived and designed the experiment. CP performed experiments and all the analyses. DE and TB provided bioinformatics support. $\mathrm{CP}$ analysed all the results with the help of HJvE and JHV. CP wrote the manuscript. JHV, RGFV and HJvE helped to write, improve and review the latest version of this manuscript. All authors read and approved the final manuscript.

\section{Funding}

This work was performed in a project called "An integrated genomics and effectoromics impulse for potato wart resistance management and breeding" which was financially supported by the Dutch Topsector Horticulture \& Starting Materials. Within the Topsector, private industry, knowledge institutes and the government are working together on innovations for sustainable production of safe and healthy food and the development of a healthy green environment. CP was supported by a Walsh fellowship provided by Teagasc.

\section{Availability of data and materials}

The datasets used and/or analysed during the current study are available in the published manuscript or available from the corresponding author on reasonable request.

\section{Ethics approval and consent to participate}

Not applicable.

\section{Consent for publication}

Not applicable.

\section{Competing interests}

The authors declare that they have no competing interests.

Received: 18 January 2019 Accepted: 23 May 2019

Published online: 29 May 2019

\section{References}

1. Giovannoni JJ, Wing RA, Ganal MW, Tanksley SD. Isolation of molecular markers from specific chromosomal intervals using DNA pools from existing mapping populations. Nucl Acids Res. 1991;19(23):6553-68.

2. Michelmore RW, Paran I, Kesseli RV. Identification of markers linked to disease-resistance genes by bulked segregant analysis: a rapid method to detect markers in specific genomic regions by using segregating populations. Proc Natl Acad Sci. 1991;88(21):9828-32.

3. Quarrie $S A$, Lazić-Jančić $V$, Kovačević $D$, Steed A, Pekić $S$. Bulk segregant analysis with molecular markers and its use for improving drought resistance in maize. J Exp Bot. 1999;50(337):1299-306.

4. Becker A, Chao D-Y, Zhang X, Salt DE, Baxter I. Bulk segregant analysis using single nucleotide polymorphism microarrays. PLoS One. 2011;6(1):e15993.

5. Schneeberger K, Ossowski S, Lanz C, Juul T, Petersen AH, Nielsen KL, et al. SHOREmap: simultaneous mapping and mutation identification by deep sequencing. Nat Methods. 2009;1 (6):550

6. Liu S, Yeh C-T, Tang HM, Nettleton D, Schnable PS. Gene mapping via bulked segregant RNA-Seq (BSR-Seq). PLoS One. 2012;7(5):e36406.
7. Jupe F, Witek K, Verweij W, Śliwka J, Pritchard L, Etherington GJ, et al. Resistance gene enrichment sequencing (RenSeq) enables reannotation of the NB-LRR gene family from sequenced plant genomes and rapid mapping of resistance loci in segregating populations. Plant J. 2013;76(3):530-44.

8. Baird NA, Etter PD, Atwood TS, Currey MC, Shiver AL, Lewis ZA, et al. Rapid SNP discovery and genetic mapping using sequenced RAD markers. PLoS One. 2008;3(10):e3376.

9. Hartwig B, James GV, Konrad K, Schneeberger K, Turck F. Fast isogenic mapping-by-sequencing of ethyl methanesulfonate-induced mutant bulks. Plant Physiol. 2012;160(2):591-600.

10. Montenegro JD, Golicz AA, Bayer PE, Hurgobin B, Lee H, Chan C-KK, et al. The pangenome of hexaploid bread wheat. Plant J. 2017:90(5):1007-13.

11. Cantalapiedra CP, Contreras-Moreira B, Silvar C, Perovic D, Ordon F, Gracia $M P$, et al. A cluster of nucleotide-binding site-leucine-rich repeat genes resides in a barley powdery mildew resistance quantitative trait loci on 7HL. Plant Genome. 2016. https://doi.org/10.3835/plantgenome2015 .10 .0101$.

12. Rahman A, Hallgrímsdóttir I, Eisen M, Pachter L. Association mapping from sequencing reads using k-mers. eLife. 2018;7:e32920.

13. Arora S, Steuernagel B, Gaurav K, Chandramohan S, Long Y, Matny O, et al. Resistance gene cloning from a wild crop relative by sequence capture and association genetics. Nat Biotechnol. 2019;37(2):139-43.

14. Przetakiewicz J. The viability of winter sporangia of Synchytrium endobioticum (Schilb.) Perc. from Poland. Am J Potato Res. 2015;92(6):704-8.

15. PM 9/5 (2) Synchytrium endobioticum. EPPO Bull. 2017;47(3):511-2.

16. Hehl R, Faurie E, Hesselbach J, Salamini F, Whitham S, Baker B, et al. TMV resistance gene $\mathrm{N}$ homologues are linked to Synchytrium endobioticum resistance in potato. Theor Appl Genet. 1999;98(3):379-86.

17. Brugmans $B$, Hutten RGB, Rookmaker ANO, Visser RGF, van Eck HJ. Exploitation of a marker dense linkage map of potato for positional cloning of a wart disease resistance gene. Theor Appl Genet. 2006;112(2):269-77.

18. Plich J, Przetakiewicz J, Śliwka J, Flis B, Wasilewicz-Flis I, ZimnochGuzowska E. Novel gene Sen2 conferring broad-spectrum resistance to synchytrium endobioticum mapped to potato chromosome XI. Theor Appl Genet. 2018. https://doi.org/10.1007/s00122-018-3154-y.

19. Ballvora A, Flath K, Lübeck J, Strahwald J, Tacke E, Hofferbert H-R, et al. Multiple alleles for resistance and susceptibility modulate the defense response in the interaction of tetraploid potato (Solanum tuberosum) with Synchytrium endobioticum pathotypes 1, 2,6 and 18. Theor Appl Genet. 2011;123(8):1281-92.

20. Groth J, Song Y, Kellermann A, Schwarzfischer A. Molecular characterisation of resistance against potato wart races 1, 2, 6 and 18 in a tetraploid population of potato (Solanum tuberosum subsp. tuberosum). J Appl Genet. 2013;54(2):169-78.

21. Obidiegwu JE, Sanetomo R, Flath K, Tacke E, Hofferbert H-R, Hofmann A, et al. Genomic architecture of potato resistance to Synchytrium endobioticum disentangled using SSR markers and the 8.3k SolCAP SNP genotyping array. BMC Genet. 2015;16(1):38.

22. Bartkiewicz A, Chilla F, Terefe-Ayana D, Lübeck J, Strahwald J, Tacke E, et al. Improved genetic resolution for linkage mapping of resistance to potato wart in monoparental dihaploids with potential diagnostic value in tetraploid potato varieties. Theor Appl Genet. 2018. https://doi.org/10.1007/ s00122-018-3172-9.

23. JKI (2018). https://pflanzengesundheit.julius-kuehn.de/dokumente/uploa d/29991_bm-2018resistente_kartoffelsorten.pdf. Accessed 15 Oct 2018.

24. Glynne MD. Infection experiments with wart disease of potatoes synchytrium endobioticum (Schilb.) Perc. Ann Appl Biol. 1925;12(1):34-60.

25. Lemmerzahl J. A new simplified method of inoculation of potato cultivars to test for wart resistance. Züchter. 1930;2:288-97.

26. Fulton TM, Chunwongse J, Tanksley SD. Microprep protocol for extraction of DNA from tomato and other herbaceous plants. Plant Mol Biol Rep. 1995;13(3):207-9.

27. Murray MG, Thompson WF. Rapid isolation of high molecular weight plant DNA. Nucl Acids Res. 1980;8(19):4321-5.

28. Bolger AM, Lohse M, Usadel B. Trimmomatic: a flexible trimmer for illumina sequence data. Bioinformatics. 2014;30(15):2114-20.

29. Kaplinski L, Lepamets M, Remm M. GenomeTester4: a toolkit for performing basic set operations - union, intersection and complement on k-mer lists. GigaScience. 2015. https://doi.org/10.1186/s13742-015-0097-y. 
30. Li H, Durbin R. Fast and accurate short read alignment with BurrowsWheeler transform. Bioinformatics. 2009;25(14):1754-60.

31. Potato Genome Sequencing Consortium, Pan S, Cheng S, Zhang B, Mu D, Ni P, et al. Genome sequence and analysis of the tuber crop potato. Nature. 2011:475(7355):189-95.

32. Sharma SK, Bolser D, de Boer J, Sønderkær M, Amoros W, Carboni MF, et al. Construction of reference chromosome-scale pseudomolecules for potato: integrating the potato genome with genetic and physical maps. G3 Genes Genomes Genet. 2013;3(11):2031-47.

33. Quinlan AR, Hall IM. BEDTools: a flexible suite of utilities for comparing genomic features. Bioinformatics. 2010;26(6):841-2

34. Bankevich A, Nurk S, Antipov D, Gurevich AA, Dvorkin M, Kulikov AS, et al. SPAdes: a new genome assembly algorithm and its applications to single-cell sequencing. J Comput Biol. 2012;19(5):455-77.

35. Li H, Handsaker B, Wysoker A, Fennell T, Ruan J, Homer N, et al. The sequence alignment/map format and SAMtools. Bioinformatics. 2009;25(16):2078-9.

36. Tarasov A, Vilella AJ, Cuppen E, Nijman IJ, Prins P. Sambamba: fast processing of NGS alignment formats. Bioinformatics. 2015;31(12):2032-4.

37. Rozen S, Skaletsky H. Primer3 on the WWW for general users and for biologist programmers. In: Krawetz S, Misener S, editors. Bioinformatics methods and protocols: methods in molecular biology. Totowa: Humana Press; 2000. p. 365-86

38. Bakker E, Borm T, Prins P, van der Vossen E, Uenk G, Arens M, et al. A genome-wide genetic map of NB-LRR disease resistance loci in potato. Theor Appl Genet. 2011;123(3):493-508.

39. Vossen JH, Dezhsetan S, Esselink D, Arens M, Sanz MJ, Verweij W, et al. Novel applications of motif-directed profiling to identify disease resistance genes in plants. Plant Methods. 2013;9(1):37.

40. Bukasov SM, Kameraz AJ. Bases of potato breeding. Moskva: Gosudarstvennoe Izdatel'stvo Sel'skohozjajstvennoj Literatuny; 1959. p. 528.
41. Ross H. Potato breeding — problems and perspectives. Suppl. to J. Plant Breeding. Berlin: Paul Parey; 1986.

42. Murphy AM, De Jong H, Proudfoot KG, Proudfoot KG. A multiple disease resistant potato clone developed with classical breeding methodology. Can J Plant Pathol. 1999;21(3):207-12.

43. Nordström KJV, Albani MC, James GV, Gutjahr C, Hartwig B, Turck F, et al. Mutation identification by direct comparison of whole-genome sequencing data from mutant and wild-type individuals using $\mathrm{k}$-mers. Nat Biotechnol. 2013:31(4):325-30.

44. Audano PA, Ravishankar S, Vannberg FO. Mapping-free variant calling using haplotype reconstruction from k-mer frequencies. Bioinformatics. 2018;34(10):1659-65.

45. Gardner SN, Hall BG. When whole-genome alignments just won't work: kSNP v2 software for alignment-free SNP discovery and phylogenetics of hundreds of microbial genomes. PLoS One. 2013;8(12):e81760.

46. Pajuste F-D, Kaplinski L, Möls M, Puurand T, Lepamets M, Remm M. FastGT: an alignment-free method for calling common SNVs directly from raw sequencing reads. Sci Rep. 2017;7(1):2537.

47. Steuernagel B, Jupe F, Witek K, Jones JDG, Wulff BBH. NLR-parser: rapid annotation of plant NLR complements. Bioinformatics. 2015;31(10):1665-7.

48. Witek K, Jupe F, Witek Al, Baker D, Clark MD, Jones JDG. Accelerated cloning of a potato late blight-resistance gene using RenSeq and SMRT sequencing. Nat Biotechnol. 2016;34(6):656-60.

49. van Berloo R, Hutten RCB, van Eck HJ, Visser RGF. An online potato pedigree database resource. Potato Res. 2007;50(1):45-57.

\section{Publisher's Note}

Springer Nature remains neutral with regard to jurisdictional claims in published maps and institutional affiliations.
Ready to submit your research? Choose BMC and benefit from:

- fast, convenient online submission

- thorough peer review by experienced researchers in your field

- rapid publication on acceptance

- support for research data, including large and complex data types

- gold Open Access which fosters wider collaboration and increased citations

- maximum visibility for your research: over $100 \mathrm{M}$ website views per year

At BMC, research is always in progress.

Learn more biomedcentral.com/submissions 